\title{
Two-Stage Beamformer Design for Massive MIMO Downlink By Trace Quotient Formulation
}

\author{
Donggun Kim, Student Member, IEEE, Gilwon Lee, Student Member, IEEE, and \\ Youngchul Sung, Senior Member, IEEE
}

\begin{abstract}
In this paper, the problem of outer beamformer design based only on channel statistic information is considered for two-stage beamforming for multi-user massive MIMO downlink, and the problem is approached based on signal-to-leakage-plusnoise ratio (SLNR). To eliminate the dependence on the instantaneous channel state information, a lower bound on the average SLNR is derived by assuming zero-forcing (ZF) inner beamforming, and an outer beamformer design method that maximizes the lower bound on the average SLNR is proposed. It is shown that the proposed SLNR-based outer beamformer design problem reduces to a trace quotient problem (TQP), which is often encountered in the field of machine learning. An iterative algorithm is presented to obtain an optimal solution to the proposed TQP. The proposed method has the capability of optimally controlling the weighting factor between the signal power to the desired user and the interference leakage power to undesired users according to different channel statistics. Numerical results show that the proposed outer beamformer design method yields significant performance gain over existing methods.
\end{abstract}

Index Terms-Massive MIMO systems, two-stage beamforming, signal-to-leakage-plus-noise ratio (SLNR), trace quotient problem (TQP), adaptive weighting factor.

\section{INTRODUCTION}

$\mathbf{T}$ HE multiple-input multiple-output (MIMO) technology has prevailed in wireless communications for more than a decade. The technology has been adopted in many wireless standards since it improves the spectral efficiency and reliability of wireless communication without requiring additional bandwidth. Recently, the MIMO technology based on largescale antenna arrays at base stations, so-called massive MIMO, is considered to further improve the system performance for upcoming $5 \mathrm{G}$ wireless systems and vigorous research is being performed on this topic. Massive MIMO can support high data rates and energy efficiency and simplify receiver processing based on the asymptotic orthogonality among user channels

Manuscript received November 25, 2014; revised March 20, 2015 and April 30, 2015; accepted April 30, 2015. Date of publication May 5, 2015; date of current version June 12, 2015. This research was supported in part by Basic Science Research Program through the National Research Foundation of Korea (NRF) funded by the Ministry of Education (2013R1A1A2A10060852). This work was also supported by 'The Cross-Ministry Giga KOREA Project' of The Ministry of Science, ICT and Future Planning, Korea [GK14N0100, 5G mobile communication system development based on mmWave]. The associate editor coordinating the review of this paper and approving it for publication was M. Matthaiou.

The authors are with Department of Electrical Engineering, Korea Advanced Institute of Science and Technology (KAIST), Daejeon 305-701, Korea (e-mail: dg.kim@kaist.ac.kr; gwlee@kaist.ac.kr; ysung@ee.kaist.ac.kr).

Digital Object Identifier 10.1109/TCOMM.2015.2429646 based on large antenna arrays [1], [2]. However, realizing the benefits of massive MIMO in practical systems faces several challenges especially in widely-used frequency division duplexing (FDD) scenarios. In contrast to current small-scale MIMO systems, downlink channel estimation is a difficult problem for FDD massive MIMO systems since the number of available training symbols required for downlink channel estimation is limited by the channel coherence time and the number of channel parameters to estimate is very large [3]-[6]. Furthermore, channel state information (CSI) feedback overhead for downlink user scheduling for massive FDD multi-user MIMO can be overwhelming without some smart structure on massive MIMO systems. To overcome these difficulties associated with massive MIMO, two-stage beamforming for massive MIMO under the name of "Joint Spatial Division and Multiplexing (JSDM)" has been studied in [7]-[11]. The two-stage beamforming idea is basically a divide-and-conquer approach, and the key ideas of the two-stage beamforming strategy are 1) to partition the user population supported by the serving base station into multiple groups each with approximately the same channel covariance matrix (this can be viewed as virtual sectorization) and 2) to decompose the MIMO beamformer at the base station into two steps: an outer beamformer and an inner beamformer, as shown in Fig. 1. The outer beamformer faces the antenna array and roughly distinguishes different groups by bolstering in-group transmit power and suppressing inter-group interference, and the inner beamformer views the product of the actual channel and the outer beamformer as an effective channel, separates the users within a group, and provides spatial multiplexing among in-group users [7]. Here, major complexity reduction results from the approach that the outer beamformer is properly designed based only on channel statistic information not on CSI. In this case, the actually required CSI for the inner beamformer adopting typical zero-forcing $(\mathrm{ZF})$ or regularized ZF (RZF) beamforming is significantly reduced since it only requires the CSI of the effective channel with significantly reduced dimensions.

Several researchers followed the aforementioned framework for two-stage beamforming for massive MIMO. They adopted linear beamforming such as ZF for the inner beamformer and tackled the problem of outer beamformer design based on channel statistic information [7], [10], [11]. In [7], Adhikary et al. proposed a simple block diagonalization (BD) algorithm for the outer beamformer design, which obtains the outer beamformer by projecting the dominant eigenvectors of the desired group channel covariance matrix onto the null 


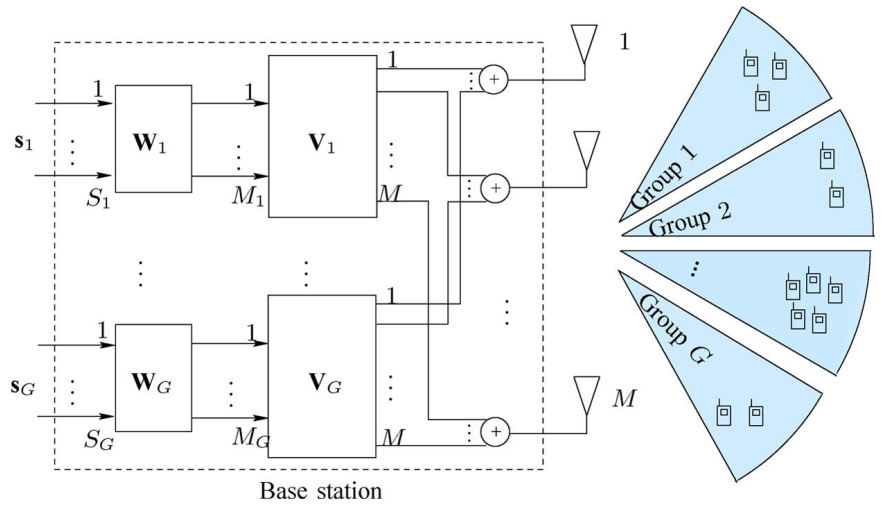

Fig. 1. Multi-group massive MIMO downlink with two-stage beamforming [9].

space of the dominant eigenspace of all other group channel covariance matrices. In [10], Chen and Lau considered the outer beamformer design criterion of minimizing the total intergroup interference power minus the weighted total desired group signal power. In this case, for a given weighting value between the total inter-group interference power and the total in-group signal power, the outer beamformer is given by a set of dominant eigenvectors of the weighted difference between the total undesired group channel covariance matrix sum and the desired group channel covariance matrix. In [11], Liu and Lau considered the outer beamformer design from a fairness perspective. In this work, they designed the outer beamformer by choosing a set of columns from a discrete Fourier transform (DFT) matrix to maximize the minimum average rate among all the users.

In this paper, we also consider the outer beamformer design based only on channel statistic information for the aforementioned two-stage beamforming framework for massive MIMO. As already shown in the previous works, computation of the signal-to-interference-plus-noise-ratio (SINR) for each receiver is difficult in this downlink scenario with interfering groups. To circumvent this difficulty, as our design criterion we adopt the average signal-to-leakage-plus-noise ratio (SLNR) criterion [12], which is shown to be Pareto-optimal in the achievable rate region in certain interference channel cases [13], [14], and propose an average SLNR-based outer beamformer design framework in single cell massive MIMO systems. ${ }^{1}$ The signal power to the desired receiver and the leakage power to other undesired receivers by the transmitter required for the SLNR method cannot be computed by considering only the outer beamformer. Instead, both the outer beamformer and the inner beamformer should jointly be considered to derive the two quantities. Thus, to simplify analysis we assume a ZF beamformer with equal power allocation for the inner beamformer although simulation is performed for both $\mathrm{ZF}$ and regularized ZF (RZF) inner beamformers. Even with this assumption of $\mathrm{ZF}$ for the inner beamformer, the derivation of average SLNR is not straightforward due to the joint nature. Thus, exploiting the fact that ZF is used for the inner beamformer, we derive a

\footnotetext{
${ }^{1}$ The multi-cell scenario considered in [10] can be cast into this single-cell multi-group setting simply by considering each base station in the multi-cell case as one group in the single-cell multi-group case.
}

lower bound on the average SLNR that is a function of only channel statistics and the outer beamformer, and our design criterion is to maximize this lower bound on the average SLNR under the constraint that the outer beamformer matrix has orthonormal columns. ${ }^{2}$ Then, we cast this constrained optimization problem as a trace quotient problem (TQP), which is often encountered in the field of pattern recognition, computer vision, and machine learning [15]-[17]. To obtain an optimal solution to the formulated TQP, we modify the algorithm in [17] to fit into the considered case and show the optimality and convergence of the modified algorithm based on existing results [15]-[17]. Numerical results show that the proposed outer beamformer design approach yields significant sum rate gain over the existing algorithms in [7] and [10].

Notation and Organization: We will make use of standard notational conventions. Vectors and matrices are written in boldface with matrices in capitals. All vectors are column vectors. For a matrix $\mathbf{X}, \mathbf{X}^{*}, \mathbf{X}^{T}, \mathbf{X}^{H},[\mathbf{X}]_{i, j}$, and $\operatorname{Tr}(\mathbf{X})$ indicate the complex conjugate, transpose, conjugate transpose, $(i, j)$-th element, and trace of $\mathbf{X}$, respectively. $\mathbf{I}_{n}$ stands for the identity matrix of size $n$ (the subscript is omitted when unnecessary). For vector $\mathbf{x},\|\mathbf{x}\|$ represents the 2-norm of $\mathbf{x}$. diag $\left(x_{1}, x_{2}, \cdots, x_{n}\right)$ means a diagonal matrix with diagonal entries $x_{1}, x_{2}, \cdots, x_{n}$. The notation $\mathbf{x} \sim \mathcal{C N}(\boldsymbol{\mu}, \boldsymbol{\Sigma})$ means that $\mathbf{x}$ is complex circularly-symmetric Gaussian distributed with mean vector $\boldsymbol{\mu}$ and covariance matrix $\boldsymbol{\Sigma} . \mathbb{E}\{\cdot\}$ denotes the expectation. $\iota \triangleq \sqrt{-1}$ and $\mathbb{C}$ is the set of complex numbers.

The remainder of this paper is organized as follows. The system model is described in Section II. In Section III, a lower bound on the average SLNR is derived and the outer beamformer design problem is formulated as a TQP. An iterative algorithm for the TQP is presented and its optimality and convergence are shown. The performance of the proposed algorithm is investigated in Section IV, followed by the conclusion in Section V.

\section{SyStem Model}

We consider a single-cell massive MIMO downlink system in which a base station with a uniform linear array (ULA) of $M$ transmit antennas serves $K$ single-antenna users. We assume that $K$ users in the cell are partitioned into $G$ groups such that $K=\sum_{g=1}^{G} K_{g}$, where $K_{g}$ is the number of users in group $g$, and $K_{g}$ users in group $g$ have the same $M \times M$ channel covariance matrix $\mathbf{R}_{g}$ as in [7], [9]-[11]. We assume a typical spatial correlation channel model [4], [18]. That is, the channel of user $k$ in group $g$ is given by

$$
\mathbf{h}_{g_{k}}=\mathbf{R}_{g}^{1 / 2} \mathbf{h}_{g_{k}}^{i . i . d},
$$

where $\mathbf{h}_{g_{k}}^{\text {i.i.d }} \in \mathbb{C}^{M \times 1} \stackrel{\text { i.i.d. }}{\sim} \mathcal{C N}\left(\mathbf{0}, \mathbf{I}_{M}\right)$. We assume that the outer beamformer implements virtual sectorization, i.e., the outer

\footnotetext{
${ }^{2}$ The outer beamformer matrix's having orthonormal columns is very desirable for effective downlink channel estimation [3] and downlink user scheduling [8], [9] purposes under the two-stage beamforming framework for massive MIMO.
} 
beamformer divides the overall azimuthal angle (typically 120 degrees in conventional cellular networks) into multiple virtual sectors with roughly ${ }^{3} 2 \Delta$ degrees for azimuthal coverage for each virtual sector. A channel model considering such a situation is the one-ring scattering model [19], [20], which captures the base station's elevation and local scattering around the users. Under this model, the channel covariance matrix for each sector covering $2 \Delta$ azimuthal angle centered at $\theta$ can be precomputed as [19], [20]

$$
\left[\mathbf{R}_{g}\right]_{k, l}=\frac{1}{2 \Delta} \int_{\theta-\Delta}^{\theta+\Delta} e^{-l 2 \pi(k-l) \frac{d}{\lambda_{c}} \sin \omega} d \omega
$$

where $\lambda_{c}$ is the carrier wavelength, $d$ is the antenna spacing, $\theta$ is the angle of the center of the subsector, and $\Delta$ is the angle spread (AS). From here on, we will assume that the channel covariance matrices $\mathbf{R}_{g}, g=1, \cdots, G$ are given to the base station.

As in [7]-[11], we consider two-stage beamforming for downlink transmission with outer beamformers $\left\{\mathbf{V}_{g}, g=\right.$ $1, \cdots, G\}$ for group separation or virtual sectorization and an inner beamformer $\mathbf{W}_{g}$ for user separation within group $g$ for each $g=1, \cdots, G$, as shown in Fig. 1. Denote the overall $K \times M$ channel matrix as $\mathbf{H}=\left[\mathbf{H}_{1}^{H}, \mathbf{H}_{2}^{H}, \cdots, \mathbf{H}_{G}^{H}\right]^{H}$, where $\mathbf{H}_{g}=\left[\mathbf{h}_{g_{1}}, \mathbf{h}_{g_{2}}, \cdots, \mathbf{h}_{g_{K_{g}}}\right]^{H}$ is the $K_{g} \times M$ channel matrix for the users in group $g$. Then, the signal vector received by all the users in the cell is given by

$$
\mathbf{y}=\mathbf{H V W s}+\mathbf{n},
$$

where the overall outer beamformer matrix $\mathbf{V}$ is partitioned into $G$ submatrices as $\mathbf{V}=\left[\mathbf{V}_{1}, \mathbf{V}_{2}, \cdots, \mathbf{V}_{G}\right]$ with $\mathbf{V}_{g} \in \mathbb{C}^{M \times M_{g}}$ satisfying ${ }^{4}$

$$
\mathbf{V}_{g}^{H} \mathbf{V}_{g}=\mathbf{I}
$$

the overall inner beamformer $\mathbf{W}$ has a block diagonal structure as $\mathbf{W}=\operatorname{diag}\left(\mathbf{W}_{1}, \mathbf{W}_{2}, \cdots, \mathbf{W}_{G}\right)$ with the inner beamformer $\mathbf{W}_{g}=\left[\mathbf{w}_{g_{1}}, \mathbf{w}_{g_{2}}, \cdots, \mathbf{w}_{g_{g}}\right] \in \mathbb{C}^{M_{g} \times S_{g}}$ for group $g$; $\mathbf{s}=\left[\mathbf{s}_{1}^{H}, \mathbf{s}_{2}^{H}, \ldots, \mathbf{s}_{g}^{H}\right]^{H} \sim \mathcal{C N}\left(\mathbf{0}, \mathbf{I}_{S}\right)$ is the data vector with $\mathbf{s}_{g} \sim \mathcal{C N}\left(\mathbf{0}, \mathbf{I}_{S_{g}}\right) ;$ and $\mathbf{n}=\left[\mathbf{n}_{1}^{H}, \mathbf{n}_{2}^{H}, \cdots, \mathbf{n}_{G}^{H}\right]^{H} \sim \mathcal{C N}\left(\mathbf{0}, \sigma^{2} \mathbf{I}_{K}\right)$ is the noise vector. Thus, $M_{g}$ is the dimension of the effective MIMO channel seen by the inner beamformer $\mathbf{W}_{g}$, and $S_{g}$ is the number of data streams for group $g$. We assume that the base station has an average transmit power constraint as $\mathbb{E}\left[\operatorname{Tr}\left(\mathbf{V W} \mathbf{s} \mathbf{s}^{H} \mathbf{W}^{H} \mathbf{V}^{H}\right)\right]=\operatorname{Tr}\left(\mathbf{V W E}\left[\mathbf{s} \mathbf{s}^{H}\right] \mathbf{W}^{H} \mathbf{V}^{H}\right)=$ $\operatorname{Tr}\left(\mathbf{V W W} \mathbf{W}^{H} \mathbf{V}^{H}\right)=\operatorname{Tr}\left(\mathbf{W} \mathbf{W}^{H}\right) \leq P_{T}$.

Combining $\mathbf{H V}$, we can rewrite the data model (3) as

$$
\mathbf{y}=\widetilde{\mathbf{H W}}+\mathbf{n},
$$

\footnotetext{
${ }^{3}$ Each sector cannot be completely separated because the number of transmit antennas is finite and there always exists some overlap among virtual subsectors, even in the case we design the main coverage angle of $2 \Delta$ of each sector to be small.

${ }^{4}$ The orthogonality constraint is desirable for random beamforming type user scheduling [8] or ReDOS-PBR user scheduling [9] for two-stage beamforming based massive MIMO.
}

where

$$
\widetilde{\mathbf{H}} \triangleq \mathbf{H V}=\left[\begin{array}{cccc}
\mathbf{H}_{1} \mathbf{V}_{1} & \mathbf{H}_{1} \mathbf{V}_{2} & \ldots & \mathbf{H}_{1} \mathbf{V}_{G} \\
\mathbf{H}_{2} \mathbf{V}_{1} & \mathbf{H}_{2} \mathbf{V}_{2} & \ldots & \mathbf{H}_{2} \mathbf{V}_{G} \\
\vdots & \vdots & \ddots & \vdots \\
\mathbf{H}_{G} \mathbf{V}_{1} & \mathbf{H}_{G} \mathbf{V}_{2} & \ldots & \mathbf{H}_{G} \mathbf{V}_{G}
\end{array}\right]
$$

and $\widetilde{\mathbf{H}}_{g} \triangleq \mathbf{H}_{g} \mathbf{V}_{g} \in \mathbb{C}^{K_{g} \times M_{g}}$ is the effective MIMO channel seen by the inner beamformer $\mathbf{W}_{g}$ for group $g$. We assume that the CSI of the effective MIMO channel $\widetilde{\mathbf{H}}_{g}, g=1, \cdots, G$, is available to the transmitter (please see [3]) and the inner beamformer $\mathbf{W}_{g} \in \mathbb{C}^{M_{g} \times S_{g}}\left(M_{g} \geq S_{g}\right)$ for each $g=1,2, \cdots, G$ is designed as a zero-forcing beamformer with equal power $\left\|\mathbf{w}_{g_{k}}\right\|^{2}=1$ for each user based on the effective CSI $\tilde{\mathbf{H}}_{g}$, i.e.,

$$
\mathbf{W}_{g}=\mathbf{V}_{g}^{H} \mathbf{H}_{g}^{H}\left(\mathbf{H}_{g} \mathbf{V}_{g} \mathbf{V}_{g}^{H} \mathbf{H}_{g}^{H}\right)^{-1} \mathbf{P}_{g},
$$

where $\mathbf{P}_{g}=\operatorname{diag}\left(p_{g_{1}}, \cdots, p_{g_{S}}\right)$, and $p_{g_{k}}$ is the transmit power scaling factor for user $k$ in group $g$ for satisfying $\left\|\mathbf{w}_{g_{k}}\right\|^{2}=1$. The received signal vector for the users in group $g$ is given by

$$
\mathbf{y}_{g}=\mathbf{H}_{g} \mathbf{V}_{g} \mathbf{W}_{g} \mathbf{s}_{g}+\sum_{g^{\prime} \neq g} \mathbf{H}_{g} \mathbf{V}_{g^{\prime}} \mathbf{W}_{g^{\prime}} \mathbf{s}_{g^{\prime}}+\mathbf{n}_{g},
$$

where $\mathbf{s}_{g}=\left[s_{g_{1}}, s_{g_{2}}, \cdots s_{g_{S_{g}}}\right]^{T} \in \mathbb{C}^{S_{g} \times 1}$ and $\mathbf{n}_{g}=\left[n_{g_{1}}, n_{g_{2}}, \cdots\right.$ $\left.n_{g_{K}}\right]^{T} \in \mathbb{C}^{K_{g} \times 1}$ are the data and noise vectors for group $g$, respectively. The received signal of user $k$ in group $g$ is given by

$$
\begin{array}{r}
y_{g_{k}}=\mathbf{h}_{g_{k}}^{H} \mathbf{V}_{g} \mathbf{w}_{g_{k}} s_{g_{k}}+\sum_{k^{\prime} \neq k} \mathbf{h}_{g_{k}}^{H} \mathbf{V}_{g} \mathbf{w}_{g_{k^{\prime}}} s_{g_{k^{\prime}}} \\
+\sum_{g^{\prime} \neq g} \sum_{j=1}^{K_{g^{\prime}}} \mathbf{h}_{g_{k}}^{H} \mathbf{V}_{g^{\prime}} \mathbf{w}_{g_{j}^{\prime}} s_{g_{j}^{\prime}}+n_{g_{k}},
\end{array}
$$

where the second and third terms in the right-hand side (RHS) of (9) are the intra-group and inter-group interference, respectively. With the assumed ZF inner beamforming the intra-group interference is completely eliminated, and the signal-tointerference-plus-noise ratio (SINR) at user $k$ in group $g$ is given by

$$
\operatorname{SINR}_{g_{k}}=\frac{\left|\mathbf{h}_{g_{k}}^{H} \mathbf{V}_{g} \mathbf{w}_{g_{k}}\right|^{2}}{\sum_{g^{\prime} \neq g} \sum_{j=1}^{K_{g^{\prime}}}\left|\mathbf{h}_{g_{k}}^{H} \mathbf{V}_{g^{\prime}} \mathbf{w}_{g_{j}^{\prime}}\right|^{2}+\sigma^{2}}
$$

Using the SINR as the optimization criterion, one could try to design the outer beamformer $\left\{\mathbf{V}_{g}, g=1, \cdots, G\right\}$ to maximize a relevant measure such as the sum rate. However, this criterion generally leads to a challenging nonconvex optimization problem since each user's SINR is jointly dependent on $\left\{\mathbf{V}_{g}, g=\right.$ $1, \cdots, G\}$ in a nonconvex manner [12], [13]. To circumvent the difficulty, we here adopt the SLNR approach proposed in [12] and [13]. 
The SLNR approach considers the ratio between the signal power to the desired receiver and the power of the total interference (to undesired receivers caused by the desired signal) plus noise, not the power of the total interference received at the desired receiver. The rationale for this approach is that it is reasonable for the transmitter to maximize the signal power to the desired receiver for a given allowed level of interference to undesired receivers in multi-user interference channels. The SLNR method is shown to be Pareto-optimal in the rate region in certain MIMO interference channel scenarios [13], [14]. Under the assumption of ZF inner beamforming with equal power allocation, the SLNR for user $k$ in group $g$ is given by

$$
\operatorname{SLNR}_{g_{k}}=\frac{\left|\mathbf{h}_{g_{k}}^{H} \mathbf{V}_{g} \mathbf{w}_{g_{k}}\right|^{2}}{\sum_{g^{\prime} \neq g} \sum_{j=1}^{K_{g^{\prime}}}\left|\mathbf{h}_{g_{j}^{\prime}}^{H} \mathbf{V}_{g} \mathbf{w}_{g_{k}}\right|^{2}+\sigma^{2}} .
$$

Note that the key difference between (10) and (11) is that the SINR of user $g_{k}$ (at the receiver side) is a joint function of $\left\{\mathbf{V}_{g}, g=1, \cdots, G\right\}$, whereas the SLNR of user $g_{k}$ (at the transmitter side) is a function of only $\mathbf{V}_{g}$ not of $\left\{\mathbf{V}_{g^{\prime}}, g^{\prime} \neq g\right\}$. Note also that the SLNR of user $g_{k}$ is a function of the channel $\left\{\mathbf{h}_{g_{k}}, \mathbf{h}_{g_{j}^{\prime}}, g^{\prime} \neq g, j=1, \cdots, K_{g^{\prime}}\right\}$ and the inner beamformer $\mathbf{w}_{g_{k}}$ in addition to $\mathbf{V}_{g}$.

\section{Outer BeAmformer Design CRITERION AND OPTIMIZATION}

Recall that the main advantage of the two-stage beamforming results from the fact that the outer beamformer is designed without knowing the CSI of the actual channel $\left\{\mathbf{h}_{g_{k}}, k=1, \cdots, K_{g}, g=1, \cdots, G\right\}$ [7]-[11]. Thus, the outer beamformer should be designed based only on the channel covariance matrices $\left\{\mathbf{R}_{g}, g=1, \cdots, G\right\}$ and this leads to using the average SLNR as our design criterion. Hence, we formulate the outer beamformer design problem as follows:

$$
\mathcal{P}_{1}: \mathbf{V}_{g}^{*}=\underset{\mathbf{V}_{g}^{H} \mathbf{V}_{g}=\mathbf{I}}{\arg \max } \sum_{k=1}^{K_{g}} \mathbb{E}\left[\mathrm{SLNR}_{g_{k}}\right]
$$

for each $g=1,2, \cdots, G$. Although Problem $\mathcal{P}_{1}$ is conceptually simple, solving the optimization problem is not straightforward. The first difficulty is the derivation of the average SLNR since the random quantities (i.e., the channel vectors) are both in the numerator and the denominator as seen in (11) and a closed-form expression of the average SLNR is not available. To circumvent this difficulty, we first derive a lower bound on the average SLNR and maximize this lower bound on the average SLNR under the constraint $\mathbf{V}_{g}^{H} \mathbf{V}_{g}=\mathbf{I}$.

For a given outer beamformer $\mathbf{V}_{g}$ and a given inner beamformer $\mathbf{W}_{g}$, the SLNR of user $g_{k}$ averaged over channel real- izations is lower bounded as follows:

$$
\begin{aligned}
& \mathbb{E}\left\{\operatorname{SLNR}_{g_{k}}\right\} \\
& =\mathbb{E}\left\{\frac{\left|\mathbf{h}_{g_{k}}^{H} \mathbf{V}_{g} \mathbf{w}_{g_{k}}\right|^{2}}{\sum_{g^{\prime} \neq g} \sum_{j=1}^{K_{g^{\prime}}}\left|\mathbf{h}_{g_{j}^{\prime}}^{H} \mathbf{V}_{g} \mathbf{w}_{g_{k}}\right|^{2}+\sigma^{2}}\right\}, \\
& \stackrel{(a)}{\geq} \mathbb{E}\left\{\frac{\left|\mathbf{h}_{g_{k}}^{H} \mathbf{V}_{g} \mathbf{w}_{g_{k}}\right|^{2}}{\sum_{g^{\prime} \neq g} \sum_{j=1}^{K_{g^{\prime}}}\left\|\mathbf{h}_{g_{j}^{\prime}}^{H} \mathbf{V}_{g}\right\|^{2}\left\|\mathbf{w}_{g_{k}}\right\|^{2}+\sigma^{2}}\right\} \text {, } \\
& \stackrel{(b)}{=} \mathbb{E}\left\{\frac{\left|\mathbf{h}_{g_{k}}^{H} \mathbf{V}_{g} \mathbf{w}_{g_{k}}\right|^{2}}{\sum_{g}^{\prime} \neq g \sum_{j=1}^{K_{g^{\prime}}}\left\|\mathbf{h}_{g_{j}^{H}}^{H} \mathbf{V}_{g}\right\|^{2}+\sigma^{2}}\right\} \text {, } \\
& \stackrel{(c)}{=} \mathbb{E}\left\{\left|\mathbf{h}_{g_{k}}^{H} \mathbf{V}_{g} \mathbf{w}_{g_{k}}\right|^{2}\right\} \mathbb{E}\left\{\frac{1}{\sum_{g^{\prime} \neq g} \sum_{j=1}^{K_{g^{\prime}}}\left\|\mathbf{h}_{g_{j}^{\prime}}^{H} \mathbf{V}_{g}\right\|^{2}+\sigma^{2}}\right\}, \\
& \stackrel{(d)}{\geq} \mathbb{E}\left\{\left|\mathbf{h}_{g_{k}}^{H} \mathbf{V}_{g} \mathbf{w}_{g_{k}}\right|^{2}\right\} \frac{1}{\mathbb{E}\left\{\sum_{g^{\prime} \neq g} \sum_{j=1}^{K_{g^{\prime}}}\left\|\mathbf{h}_{g_{j}^{\prime}}^{H} \mathbf{V}_{g}\right\|^{2}+\sigma^{2}\right\}}, \\
& =\frac{\mathbb{E}\left\{\left|\mathbf{h}_{g_{k}}^{H} \mathbf{V}_{g} \mathbf{w}_{g_{k}}\right|^{2}\right]}{\sum_{g^{\prime} \neq g} \sum_{j=1}^{K_{g^{\prime}}} \operatorname{Tr}\left(\mathbf{V}_{g}^{H} \mathbb{E}\left\{\mathbf{h}_{g_{j}^{\prime}} \mathbf{h}_{g_{j}^{\prime}}^{H}\right\} \mathbf{V}_{g}\right)+\sigma^{2}}, \\
& \stackrel{(e)}{=} \frac{\mathbb{E}\left\{\left|\mathbf{h}_{g_{k}}^{H} \mathbf{V}_{g} \mathbf{w}_{g_{k}}\right|^{2}\right\}}{\operatorname{Tr}\left(\mathbf{V}_{g}^{H} \mathbf{R}_{g, 2} \mathbf{V}_{g}\right)} \text {. }
\end{aligned}
$$

Here, (a) follows from the sub-multiplicativity of norm $\|\mathbf{A B}\| \leq$ $\|\mathbf{A}\|\|\mathbf{B}\|$; (b) follows from the equal power allocation $\left\|\mathbf{w}_{g_{k}}\right\|=1$; (c) follows from the independence between the desired-group channels and other group channels; (d) results from Jensen's inequality since the function $f(x)=\frac{1}{x}$ is convex for $x \geq 0$; and (e) follows from

$$
\mathbf{R}_{g, 2} \triangleq \sum_{g^{\prime} \neq g} K_{g^{\prime}} \mathbf{R}_{g^{\prime}}+\frac{\sigma^{2}}{M_{g}} \mathbf{I} .
$$

The next difficulty in deriving a lower bound on the average SLNR is that the ZF inner beamformer $\mathbf{w}_{g_{k}}$ is based on the CSI of the effective channel $\mathbf{H}_{g} \mathbf{V}_{g}$. However, at the time of designing $\mathbf{V}_{g}$ this effective channel is not determined. This difficulty is properly circumvented by exploiting the property of ZF inner beamforming and a lower bound on the average signal power appearing in the numerator of the RHS of (17) is given in the following theorem.

Theorem 1: When the outer beamformer $\mathbf{V}_{g}$ is given and the inner-beamformer $\mathbf{W}_{g}=\left[\mathbf{w}_{g_{1}}, \mathbf{w}_{g_{2}}, \cdots, \mathbf{w}_{g_{K_{g}}}\right]$ is a ZF beamformer with equal power allocation, $\mathbb{E}\left[\left|\mathbf{h}_{g_{k}}^{H} \mathbf{V}_{g} \mathbf{w}_{g_{k}}\right|^{2}\right]$ is lowerbounded as

$$
\mathbb{E}\left\{\left|\mathbf{h}_{g_{k}}^{H} \mathbf{V}_{g} \mathbf{w}_{g_{k}}\right|^{2}\right\} \geq \operatorname{Tr}\left(\mathbf{V}_{g}^{H} \mathbf{R}_{g} \mathbf{V}_{g}\right)-\left(K_{g}-1\right) \lambda_{g},
$$


where $\lambda_{g}$ is the largest eigenvalue of the channel covariance matrix $\mathbf{R}_{g}$ of group $g$.

Proof: See Appendix.

Applying Theorem 1 to (17), we obtain a lower bound on the average SLNR, given by

$$
\begin{aligned}
\mathbb{E}\left[\mathrm{SLNR}_{g_{k}}\right] & \geq \frac{\mathbb{E}\left[\left|\mathbf{h}_{g_{k}}^{H} \mathbf{V}_{g} \mathbf{w}_{g_{k}}\right|^{2}\right]}{\operatorname{Tr}\left(\mathbf{V}_{g}^{H} \mathbf{R}_{g, 2} \mathbf{V}_{g}\right)}, \\
& \geq \frac{\operatorname{Tr}\left(\mathbf{V}_{g}^{H} \mathbf{R}_{g} \mathbf{V}_{g}\right)-\left(K_{g}-1\right) \lambda_{g}}{\operatorname{Tr}\left(\mathbf{V}_{g}^{H} \mathbf{R}_{g, 2} \mathbf{V}_{g}\right)}, \\
& =\frac{\operatorname{Tr}\left(\mathbf{V}_{g}^{H} \mathbf{R}_{g, 1} \mathbf{V}_{g}\right)}{\operatorname{Tr}\left(\mathbf{V}_{g}^{H} \mathbf{R}_{g, 2} \mathbf{V}_{g}\right)},
\end{aligned}
$$

where $\mathbf{R}_{g, 1}$ is defined as

$$
\mathbf{R}_{g, 1} \triangleq \mathbf{R}_{g}-\frac{\left(K_{g}-1\right)}{M_{g}} \lambda_{g} \mathbf{I} .
$$

Note that the derived lower bound on the average SLNR depends only on the group index $g$ not on the user index $k$. This makes sense since we assume that each user in the same group has the same channel statistics. Finally, the proposed outer beamformer design based only on the channel statistic information $\left\{\mathbf{R}_{g}, g=1, \cdots, G\right\}$ is formulated as

$$
\mathcal{P}_{2}: \mathbf{V}_{g}^{*}=\underset{\mathbf{V}_{g}^{H} \mathbf{V}_{g}=\mathbf{I} \mathbf{I}}{\arg \operatorname{Tr}\left(\mathbf{V}_{g}^{H} \mathbf{R}_{g, 2} \mathbf{V}_{g}\right)}
$$

for each $g=1,2, \cdots, G$, where $\mathbf{R}_{g, 1}$ and $\mathbf{R}_{g, 2}$ are given by (21) and (18), respectively. Note that in Problem (22), the outer beamformer design is performed for each group separately. This is an advantage of the proposed design method; complicated joint optimization is not required.

Note that in Problem $\mathcal{P}_{2}, \mathbf{R}_{g, 1}$ is Hermitian and $\mathbf{R}_{g, 2}$ is positive definite due to the added identity matrix in $\mathbf{R}_{g, 2}$ in (18). Problem $\mathcal{P}_{2}$ maximizes the quotient (or ratio) of two traces under the constraint $\mathbf{V}_{g}^{H} \mathbf{V}_{g}=\mathbf{I}$, and is known as a trace quotient problem $(T Q P)$ or trace ratio problem (TRP), which is often encountered in linear discriminant analysis (LDA) for feature extraction and dimension reduction [15], [16]. Several research works have been performed to understand the theoretical properties of TQP and to develop numerical algorithms for TQP [15]-[17].

If we relax the orthogonality constraint $\mathbf{V}_{g}^{H} \mathbf{V}_{g}=\mathbf{I}$ for the outer beamformer, then Problem $\mathcal{P}_{2}$ reduces to the following simpler optimization problem:

$$
\mathcal{P}_{3}: \mathbf{V}_{g}^{\star}=\arg \max \frac{\operatorname{Tr}\left(\mathbf{V}_{g}^{H} \mathbf{R}_{g, 1} \mathbf{V}_{g}\right)}{\operatorname{Tr}\left(\mathbf{V}_{g}^{H} \mathbf{R}_{g, 2} \mathbf{V}_{g}\right)}
$$

for each $g=1,2, \cdots, G$. It was shown in [12] that the optimal $\mathbf{V}_{g}^{\star}$ of Problem $\mathcal{P}_{3}$ is the $M_{g}$ generalized eigenvectors corresponding to the $M_{g}$ largest generalized eigenvalues of the matrix pencil $\mathbf{R}_{g, 1}-\lambda \mathbf{R}_{g, 2}$. There exist many available fast algorithms to obtain the generalized eigenvectors of the positive definite matrix pencil $\mathbf{R}_{g, 1}-\lambda \mathbf{R}_{g, 2}$ [21], [22]. Note that the obtained generalized eigenvectors from Problem $\mathcal{P}_{3}$ do not satisfy the orthogonality constraint $\mathbf{V}_{g}^{H} \mathbf{V}_{g}=\mathbf{I}$ in general. The optimal solution $\mathbf{V}_{g}^{\star}$ of Problem $\mathcal{P}_{3}$ can be decomposed by thin singular value decomposition (SVD) as

$$
\mathbf{V}_{g}^{\star}=\boldsymbol{\Phi}_{g} \mathbf{D}_{g} \boldsymbol{\Psi}_{g},
$$

where $\boldsymbol{\Phi}_{g}$ is an $M \times M_{g}$ matrix satisfying $\boldsymbol{\Phi}_{g}^{H} \boldsymbol{\Phi}_{g}=\mathbf{I} ; \mathbf{D}_{g}$ is an $M_{g} \times M_{g}$ diagonal matrix; and $\boldsymbol{\Phi}_{g}$ is an $M_{g} \times M_{g}$ unitary matrix. By setting

$$
\mathbf{V}_{g}=\boldsymbol{\Phi}_{g},
$$

we can obtain an outer beamformer satisfying the constraint $\mathbf{V}_{g}^{H} \mathbf{V}_{g}=\mathbf{I}$. Although this approach does not necessarily yield an optimal solution to Problem $\mathcal{P}_{2}$, this solution is useful for the proposed iterative algorithm in the next section to reduce the computational complexity. Specifically, the solution (25) can be used as an initialization point for the iterative algorithm. The performance of this initialization and random initialization will be evaluated in Section IV.

\section{A. Optimal Solution to TQP}

In this section, we briefly discuss the optimal solution to the TQP $\mathcal{P}_{2}$. In Theorem 2.1 of [16], the optimal solution $\mathbf{V}_{g}^{\star}$ to the TQP $\mathcal{P}_{2}$ is known as the eigenvectors corresponding to the $M_{g}$ largest eigenvalues of

$$
\mathbf{E}_{\rho\left(\mathbf{V}_{g}\right)} \triangleq \mathbf{R}_{g, 1}-\rho\left(\mathbf{V}_{g}\right) \mathbf{R}_{g, 2},
$$

when the value $\rho\left(\mathbf{V}_{g}\right) \triangleq \frac{\operatorname{Tr}\left(\mathbf{V}_{g}^{H} \mathbf{R}_{g, 1} \mathbf{V}_{g}\right)}{\operatorname{Tr}\left(\mathbf{V}_{g}{ }^{H} \mathbf{R}_{g, 2} \mathbf{V}_{g}\right)}$ is maximized. In [16] and [23], it is also shown that $\mathcal{M}=\left\{\mathbf{V}_{g} \in \mathcal{C}^{M \times M_{g}} \mid \mathbf{E}_{\rho\left(\mathbf{V}_{g}\right)} \mathbf{V}_{g}=\right.$ $\mathbf{V}_{g} \mathbf{M}_{\mathbf{V}_{g}}$ and $\mathbf{V}_{g}^{H} \mathbf{V}_{g}=\mathbf{I}_{M_{g}}$ is the set of all Karush-Kuhn-Tucker (KKT) points of TQP $\mathcal{P}_{2}$, where $\mathbf{M}_{\mathbf{V}_{g}}=\mathbf{V}_{g}^{H} \mathbf{E}_{\rho\left(\mathbf{V}_{g}\right)} \mathbf{V}_{g}$. This implies that any KKT point $\mathbf{V}_{g}$ of the TQP $\mathcal{P}_{2}$ is an orthonormal eigenbasis of the matrix $\mathbf{E}_{\rho\left(\mathbf{V}_{g}\right)}$ with the sum of the corresponding eigenvalues zero, i.e., $\operatorname{Tr}\left(\mathbf{M}_{\mathbf{V}_{g}}\right)=0$. (It is easy to see the fact that $\operatorname{Tr}\left(\mathbf{M}_{\mathbf{V}_{g}}\right)=0$ from (26).) Note that (26) is a nonlinear eigenvalue problem since the weighting factor $\rho\left(\mathbf{V}_{g}\right)$ itself is a function of the design variable $\mathbf{V}_{g}$. It is known that there is no closed-form solution to the TQP [15]-[17]. In [16], however, it is shown that any iterative method that monotonically converges to a KKT point in $\mathcal{M}$ achieves a global maximizer $\mathbf{V}_{g}^{\star}$ for the TQP by repeatedly applying the update strategy for $\rho\left(\mathbf{V}_{g}\right)$ specified in Section IV of [16]. The update strategy is that in every iteration step, $\rho\left(\mathbf{V}_{g}\right)$ is computed as $\rho\left(\mathbf{V}_{g}^{(n)}\right)$, where $\mathbf{V}_{g}^{(n)}$ is determined as the eigenvectors corresponding to the $M_{g}$ largest eigenvalues of $\mathbf{R}_{g, 1}-\rho\left(\mathbf{V}_{g}^{(n-1)}\right) \mathbf{R}_{g, 2}$. The proof of this is based on the gradient and Hessian of the cost function and optimization theory [16]. Although the statement was proved in the case that $\mathbf{R}_{g, 1}$ and $\mathbf{R}_{g, 2}$ are real symmetric and real positivedefinite symmetric, respectively, in [16], the statement can easily be extended to the case that $\mathbf{R}_{g, 1}$ and $\mathbf{R}_{g, 2}$ are Hermitian and positive-definite, respectively. Interested readers are referred to [16].

\section{B. The Outer Beamformer Design Algorithm and Its Convergence}

In [17], an iterative algorithm was proposed under the condition that $\mathbf{R}_{g, 1}$ and $\mathbf{R}_{g, 2}$ are both real symmetric positive 
semidefinite. However, in our case, $\mathbf{R}_{g, 1}$ is Hermitian and $\mathbf{R}_{g, 2}$ is positive-definite. Thus, to apply the iterative algorithm in [17] to our case, we need slight modification of the algorithm from the real matrix case to the complex matrix case. Basically, the presented algorithm iteratively optimizes the objective function subject to the orthogonality constraint with $\rho\left(\mathbf{V}_{g}\right)$ update specified in [16].

Denote the objective function of Problem $\mathcal{P}_{2}$ at the $n$-th iteration by

$$
\rho_{n}=\frac{\operatorname{Tr}\left(\mathbf{V}_{g}^{(n-1)^{H}} \mathbf{R}_{g, 1} \mathbf{V}_{g}^{(n-1)}\right)}{\operatorname{Tr}\left(\mathbf{V}_{g}^{(n-1)^{H}} \mathbf{R}_{g, 2} \mathbf{V}_{g}^{(n-1)}\right)},
$$

where $\mathbf{V}_{g}^{(n-1)}$ is the outer beamformer at the $(n-1)$-th iteration. The outer beamformer $\mathbf{V}_{g}^{(n)}$ is updated by solving the following trace difference problem:

$$
\mathbf{V}_{g}^{(n)}=\underset{\mathbf{V}_{g}^{H} \mathbf{V}_{g}=\mathbf{I}}{\arg \max } \operatorname{Tr}\left(\mathbf{V}_{g}{ }^{H}\left(\mathbf{R}_{g, 1}-\rho_{n} \mathbf{R}_{g, 2}\right) \mathbf{V}_{g}\right),
$$

where $\rho_{n}$ is given by (27). The outer beamformer $\mathbf{V}_{g}^{(n)}$ of the trace difference problem in the $n$-th iteration is updated by the $M_{g}$ eigenvectors corresponding to the $M_{g}$ largest eigenvalues of the Hermitian matrix $\mathbf{R}_{g, 1}-\rho_{n} \mathbf{R}_{g, 2}$ for given $\rho_{n}$. This procedure is iterated until the iteration converges. The proposed algorithm for Problem $\mathcal{P}_{2}$ is summarized in Algorithm 1. Note that in the proposed algorithm the initialization of $\mathbf{V}_{g}^{(0)}$ is done based on (25). This initialization significantly reduces the required number of iterations compared to random initialization.

The monotonic increase of the objective function $\rho_{n}$ by Algorithm 1 is guaranteed by the following theorem:

Algorithm 1 Outer BeamformerDesign by TQP Based on [17]

Require: The channel covariance matrices $\left\{\mathbf{R}_{g}, g=1, \cdots, G\right\}$, the noise variance $\sigma^{2}$, and the stopping tolerance $\epsilon>0$. Construct $\mathbf{R}_{g, 1}$ and $\mathbf{R}_{g, 2}$ by (21) and (18).

Set $\rho_{-1}=-\infty$ and initialize $\mathbf{V}_{g}^{(0)}$ by (25).

for $n=1,2, \cdots$ do

Compute the trace ratio $\rho_{n}$ from $\mathbf{V}_{g}^{(n-1)}$ :

$$
\rho_{n}=\frac{\operatorname{Tr}\left(\mathbf{V}_{g}^{(n-1)^{H}} \mathbf{R}_{g, 1} \mathbf{V}_{g}^{(n-1)}\right)}{\operatorname{Tr}\left(\mathbf{V}_{g}^{(n-1)^{H}} \mathbf{R}_{g, 2} \mathbf{V}_{g}^{(n-1)}\right) .}
$$

Solve the trace difference problem

$$
\mathbf{V}_{g}^{(n)}=\underset{\mathbf{V}_{g}^{H} \mathbf{V}_{g}=\mathbf{I}}{\arg \max } \operatorname{Tr}\left(\mathbf{V}_{g}{ }^{H}\left(\mathbf{R}_{g, 1}-\rho_{n} \mathbf{R}_{g, 2}\right) \mathbf{V}_{g}\right) .
$$

That is, update $\mathbf{V}_{g}^{(n)}=\left[\mathbf{v}_{g, 1}^{(n)}, \mathbf{v}_{g, 2}^{(n)}, \cdots, \mathbf{v}_{g, M_{g}}^{(n)}\right]$ by solving

$$
\left(\mathbf{R}_{g, 1}-\rho_{n} \mathbf{R}_{g, 2}\right) \mathbf{v}_{g, k}^{(n)}=v_{k}^{(n)} \mathbf{v}_{g, k}^{(n)},
$$

where $\mathbf{v}_{g, k}^{(n)}$ is the eigenvector corresponding to the $k$-th largest eigenvalue $v_{k}^{(n)}$ of $\mathbf{R}_{g, 1}-\rho_{n} \mathbf{R}_{g, 2}$.

if $\left|\rho_{n}-\rho_{n-1}\right|<\epsilon$

Break the loop.

end if

end for
Theorem 2: Algorithm 1 monotonically increases the trace quotient $\rho_{n}$, i.e., $\rho_{n+1} \geq \rho_{n}$ for all $n$.

Proof: See Appendix.

Furthermore, the boundedness of the objective function $\rho_{n}$ for all $n$ can also be shown, as stated in the following theorem:

Theorem 3: Let $\psi_{1} \geq \psi_{2} \geq \cdots \geq \psi_{M}$ and $\varphi_{1} \geq \varphi_{2} \geq \cdots \geq$ $\varphi_{M}>0$ be the sorted eigenvalues of $\mathbf{R}_{g, 1}$ and $\mathbf{R}_{g, 2}(\succ 0)$, respectively. Then, the objective function of the TQP is lowerbounded and upper-bounded as follows:

$$
\begin{aligned}
& \text { i) } \sum_{k=1}^{M_{g}} \psi_{M-k} \geq 0 \text { and } \sum_{k=1}^{M_{g}} \psi_{k} \geq 0 \text { : } \\
& \frac{\sum_{k=1}^{M_{g}} \psi_{M-k}}{\sum_{k=1}^{M_{g}} \varphi_{k}} \leq \rho\left(\mathbf{V}_{g}\right) \leq \frac{\sum_{k=1}^{M_{g}} \psi_{k}}{\sum_{k=1}^{M_{g}} \varphi_{M-k}}, \\
& \text { ii) } \sum_{k=1}^{M_{g}} \psi_{M-k}<0 \text { and } \sum_{k=1}^{M_{g}} \psi_{k} \geq 0: \\
& \text { iii) } \frac{\sum_{k=1}^{M_{g}} \psi_{M-k}}{\sum_{k=1}^{M_{g}} \varphi_{M-k}} \leq \rho\left(\mathbf{V}_{g}\right) \leq \frac{\sum_{k=1}^{M_{g}} \psi_{k}}{\sum_{k=1}^{M_{g}} \varphi_{M-k}}, \\
& \quad \frac{M_{g}}{M_{g}} \text { and } \sum_{k=1}^{M^{\prime}} \psi_{k}<0: \\
& \frac{\sum_{k=1}^{M_{g}} \psi_{M-k}}{\sum_{k=1}^{M_{g}} \varphi_{M-k}} \leq \rho\left(\mathbf{V}_{g}\right) \leq \frac{\sum_{k=1}^{M_{g}} \psi_{k}}{\sum_{k=1}^{M_{g}} \varphi_{k}} .
\end{aligned}
$$

Proof: See Appendix.

Theorems 2 and 3 together imply that the proposed outer beamformer design algorithm monotonically converges by the monotone convergence theorem. The convergence of the proposed algorithm actually guarantees its global optimality. Let us first assume that the proposed algorithm converges in the $n$-th iteration, i.e., $\rho_{n+1}=\rho_{n}$. Then, the same trace difference problem (30) in the $(n+1)$-th iteration as that in the $n$-th iteration will be solved by eigenvalue decomposition. Thus, it is easy to see that the obtained solution $\mathbf{V}_{g}$ from the proposed algorithm is a KKT point in $\mathcal{M}$. This implies the local optimality of the proposed algorithm. In Theorem 1.1. of [16], it was shown that any local optimum is also a global optimum for the TQP $\mathcal{P}_{2}$. In addition to this, Algorithm 1 adopts the $\rho\left(\mathbf{V}_{g}\right)$ update strategy specified in [16]. As the optimality result in [16] explained in Section III-A, Algorithm 1 yields a global optimizer for the TQP $\mathcal{P}_{2}$.

\section{Discussion}

Now consider the difference between the proposed outer beamformer design method and the algorithm in [10]. (In Section IV, it will be seen that both algorithms perform better 
than the outer beamformer design method in [7].) The algorithm in [10] basically minimizes

$$
\left(\sum_{g^{\prime} \neq g} \mathbf{R}_{g^{\prime}}\right)-w \mathbf{R}_{g},
$$

where $w$ is an arbitrary chosen constant weighting factor. Problem (32) is equivalent to maximizing

$$
\mathbf{R}_{g}-\lambda\left(\sum_{g^{\prime} \neq g} \mathbf{R}_{g^{\prime}}\right),
$$

where $\lambda=1 / w$. Note that the first difference is that $\mathbf{R}_{g}$ and $\sum_{g^{\prime} \neq g} \mathbf{R}_{g^{\prime}}$ are respectively used in [10] instead of $\mathbf{R}_{g, 1}$ and $\mathbf{R}_{g, 2}$ used in the proposed algorithm. Thus, in the proposed algorithm there is slight change in the signal power by including the impact of $\mathrm{ZF}$ inner beamforming and the inclusion of the thermal noise in the leakage part. However, the major difference of the proposed approach from the existing method in [10] is the formulation of the trace ratio in Problem $\mathcal{P}_{2}$. As already mentioned in Section III-A, it was shown by Zhang et al. that the optimal solution of TQP $\mathcal{P}_{2}$ is the $M_{g}$ dominant eigenvectors of

$$
\mathbf{R}_{g, 1}-\rho\left(\mathbf{V}_{g}\right) \mathbf{R}_{g, 2}
$$

with $\rho\left(\mathbf{V}_{g}\right) \quad$ (= a lower bound of $\mathbb{E}\left\{\mathrm{SLNR}_{g_{k}}\right\}$ ) achieving its maximum value [16]. Thus, the proposed algorithm updates the weighting factor $\rho\left(\mathbf{V}_{g}\right)$ for different channel statistics by solving a nonlinear eigenvalue problem ${ }^{5}$ (note that the weighting factor itself is a function of the design variable $\mathbf{V}_{g}$ ), whereas the existing method solves a linear eigenvalue problem by simply fixing the weighting factor. The procedure of updating the weighting factor of the proposed outer beamformer design approach can yield significant performance gain over the existing method, as seen in Section IV.

Now consider the difference between Problem $\mathcal{P}_{2}$ and Problem $\mathcal{P}_{3}$. The optimal solution to Problem $\mathcal{P}_{3}$ is given by the set of $M_{g}$ dominant generalized eigenvectors of $\left(\mathbf{R}_{g, 1}, \mathbf{R}_{g, 2}\right)$ satisfying [12]

$$
\mathbf{R}_{g, 1} \boldsymbol{\xi}_{i}=\lambda_{i} \mathbf{R}_{g, 2} \boldsymbol{\xi}_{i}, i=1, \cdots, M_{g}
$$

where $\lambda_{1} \geq \cdots \geq \lambda_{M_{g}}$. Since $\mathbf{R}_{g, 1}$ is Hermitian and $\mathbf{R}_{g, 2}$ is

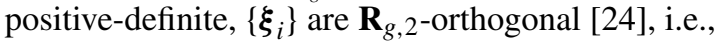

$$
\boldsymbol{\xi}_{j}^{H} \mathbf{R}_{g, 2} \boldsymbol{\xi}_{i}=0, i \neq j
$$

Thus, $\mathbf{V}_{g}=\left[\boldsymbol{\xi}_{1}, \cdots, \boldsymbol{\xi}_{M_{g}}\right]$ does not satisfy the orthogonality constraint $\mathbf{V}_{g}^{H} \mathbf{V}_{g}=\mathbf{I}$ unless $\mathbf{R}_{g, 2}=c \mathbf{I}$ for some constant $c$. However, the matrix in (34) is Hermitian and thus, it is diagonalizable by unitary similarity, i.e., it has orthonormal eigenvectors [25]. Hence, it yields an outer beamformer satisfying the orthogonality constraint. Note that (33) involves a linear Hermitian eigen-system composed of a weighted matrix difference with a fixed weighting factor and Problem $\mathcal{P}_{3}$ involves a linear

\footnotetext{
${ }^{5}$ This procedure is clearly seen in Algorithm 1.
}

generalized eigen-system (35) with a matrix pencil, whereas the proposed problem $\mathcal{P}_{2}$ involves a nonlinear Hermitian eigensystem (34) again composed of a weighted matrix difference but with a weighting factor depending on the design variable.

\section{NUMERICAL RESULTS}

In this section, we provide some numerical results to evaluate the performance of the proposed outer beamformer design method in Section III. Throughout the simulation, we considered a massive multiple-input single-output (MISO) downlink system in which a base station is equipped with a ULA of $M=$ 128 antenna elements and each of $K$ users has a single receive antenna. The $K$ users were grouped into four groups $(G=4)$, and the base station supported five users simultaneously for each group, i.e., $K_{g}=5$ for each $g=1,2,3$, and 4 . $M_{g}$ and $S_{g}$ were set as $M_{g}=M / G$ and $S_{g}=K_{g}$ for $g=1,2,3$, and 4. The channel covariance matrix for each group is specified by (2) with the center angle $\theta$ and the AS parameter $\Delta$. The channel vector for each user was independently generated according to the model (1). The stopping tolerance of the proposed outer beamformer design algorithm was set as $\epsilon=10^{-4}$. The noise power is set as $\sigma^{2}=1$. From here on, all $\mathrm{dB}$ power values are relative to $\sigma^{2}=1$.

Fig. 2(a) shows the sum rate performance of the proposed outer beamformer design algorithm and two existing algorithms in [7] and [10]. (Throughout the simulation, the weighting factor for the algorithm in [10] was set as $w=1$.) We also considered regularized ZF (RZF) [26] inner beamforming with the same outer beamformer designed by the proposed algorithm under the assumption of $\mathrm{ZF}$ inner beamforming. The regularization factor was set as $\alpha=K / P_{T}$, which is approximately optimized based on [26], [27]. The parameters for four virtual sectors covering a conventional cell sector were $\theta=$ $-45^{\circ},-15^{\circ}, 15,^{\circ}$ and $45^{\circ}$ with $\Delta=\pi / 13$ (i.e. $2 \Delta=27.7^{\circ}$ ). Note that still inter-group interference leakage comes in even though $2 \Delta<30^{\circ}$ for the channel covariance model (2) when the number of transmit antennas is finite. The power azimuth spectrum (PAS) for this setting is shown in Fig. 2(b). The curves in Fig. 2(a) is the average sum rate over 2000 independent channel realizations according to the model (1) and (2). It is seen that the proposed algorithm has significant gain over the other two algorithms. It is also seen that in the low SNR region the proposed outer beamformer combined with RZF inner beamforming has performance gain over the proposed outer beamformer combined with ZF inner beamforming as expected. Although it is not shown due to space limitation, similar relative performance is observed for different channel models such as truncated Gaussian and Laplacian power azimuth spectra.

Fig. 3 shows the sum of the total signal power to each desired group and the sum of the total leakage power to undesired groups corresponding to each group under the same setting as in Fig. 2. The shown curves are average values over 2000 channel realizations. Now the cause of the performance gain of the proposed algorithm over the existing algorithms is clear. It achieves almost the same signal power to the desired group with reduced leakage power to undesired groups compared to the two other algorithms, by solving a nonlinear eigenvalue 


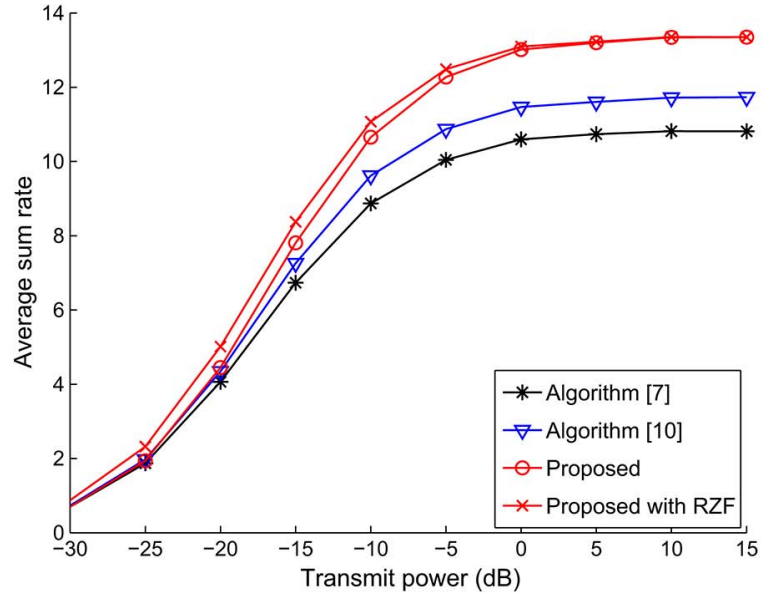

(a)

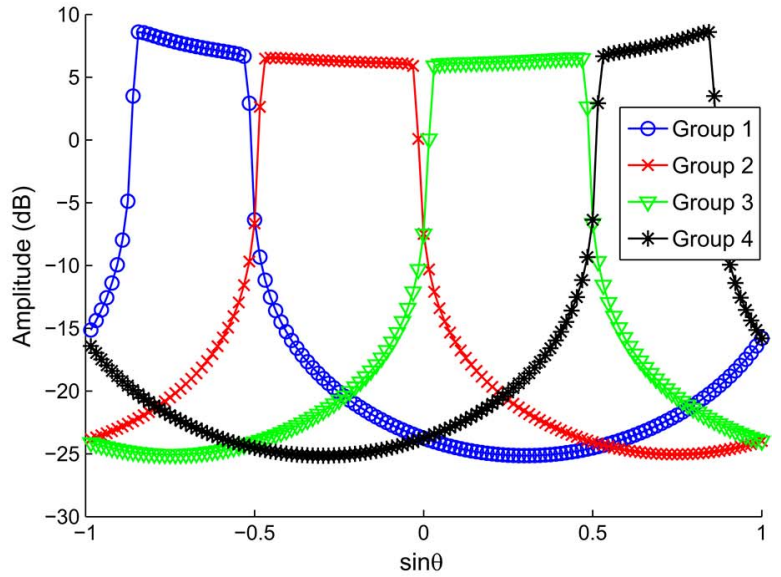

(b)

Fig. 2. $M=128, G=4, M_{g}=32, K_{g}=S_{g}=5, \Delta=\pi / 13$, and $\theta=-45^{\circ},-15^{\circ}, 15,^{\circ}$ and $45^{\circ}$ : (a) average sum rate versus transmit power and (b) PAS of the model (2). (Note that the $\mathrm{dB}$ transmit power in the $\mathrm{x}$-axis in Fig. (2)(a) is not the received SNR because of the channel gain and the beamforming gain.)

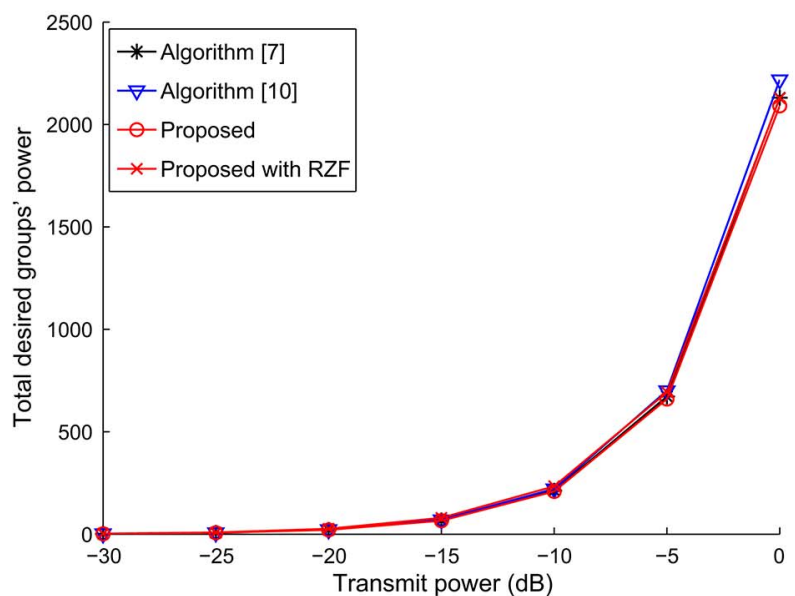

(a)

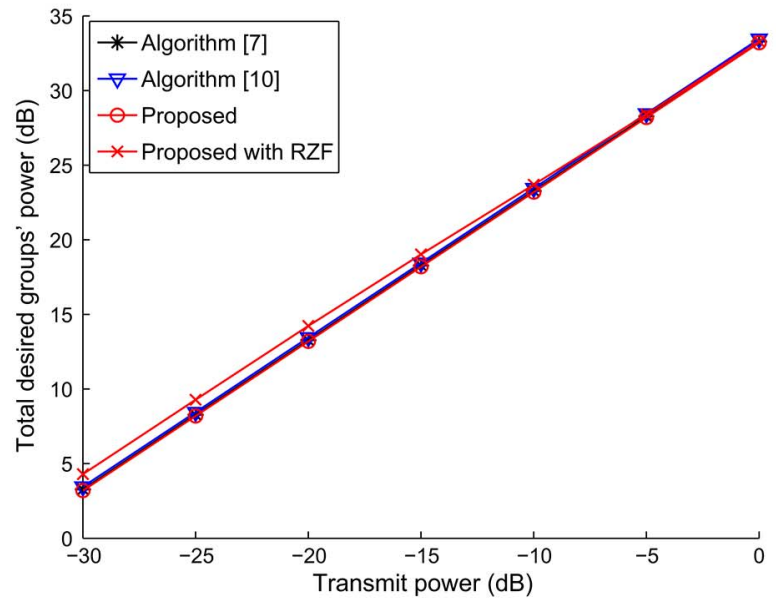

(c)

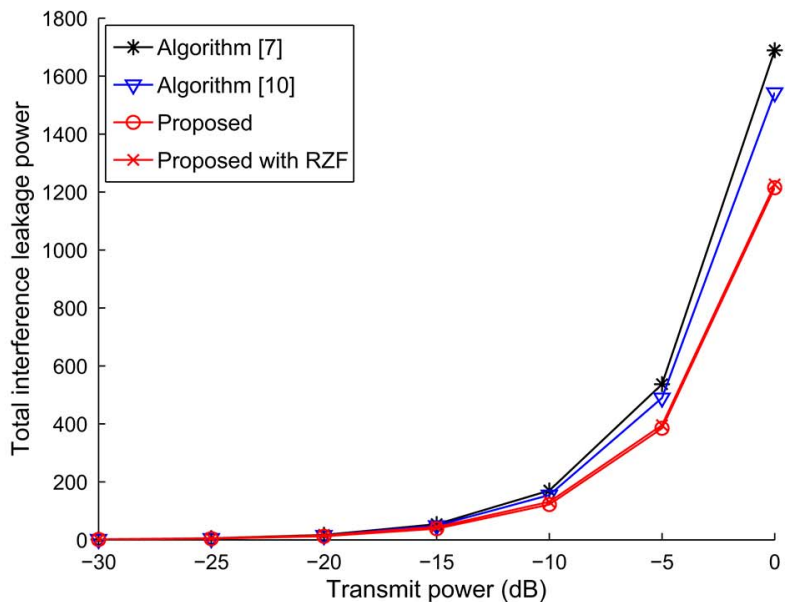

(b)

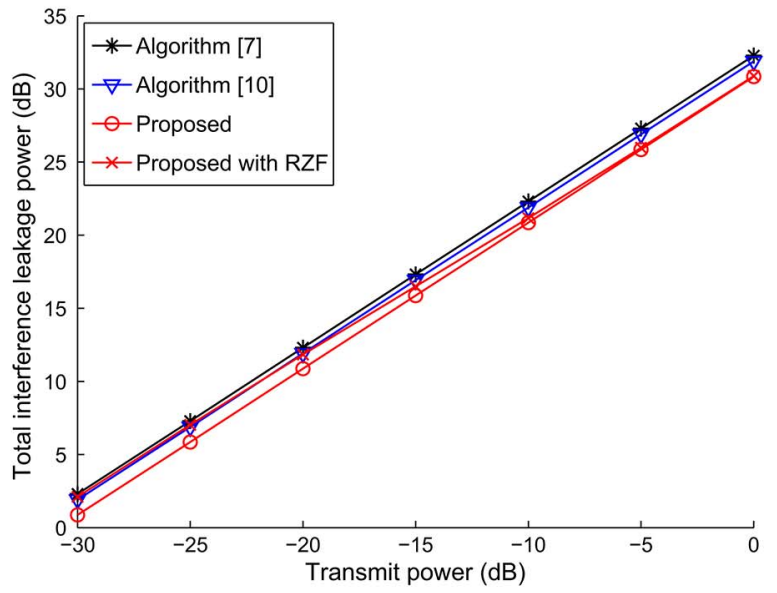

(d)

Fig. 3. (a) Total desired groups' power versus transmit power, (b) total interference leakage power versus transmit power, (c) total desired groups' power in a $\mathrm{dB}$ scale versus transmit power, and (d) total interference leakage power in a dB scale versus transmit power $\left(M=128, G=4, M_{g}=32, K_{g}=S_{g}=5, \Delta=\pi / 13\right.$, and $\theta=-45^{\circ},-15^{\circ}, 15^{\circ}$ and $45^{\circ}$ ).

problem with adaptive weighting resulting from the trace quotient formulation for the signal power and the leakage power. In the case of the algorithm [10], the performance can change with respect to the weighting factor between the desired-group signal power and the inter-group interference power. However, the optimal weighting factor depending on the channel statistics is not known a priori in the case of a constant weighting factor, and the weight factor is not adaptively optimized according to the given channel statistic information in [10]. Thus, we evaluated the sum rate performance for different channel covariance 


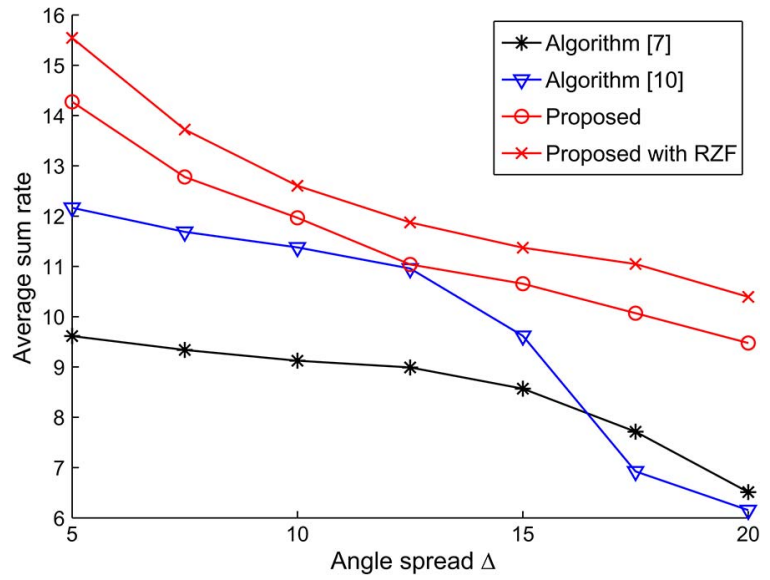

Fig. 4. Average sum rate over various algorithms versus angle spread $(M=$ $128, G=4, M_{g}=32, K_{g}=S_{g}=5, P_{T}=-10 \mathrm{~dB}$, and $\theta=-45^{\circ},-15^{\circ}$, $15,^{\circ}$ and $\left.45^{\circ}\right)$

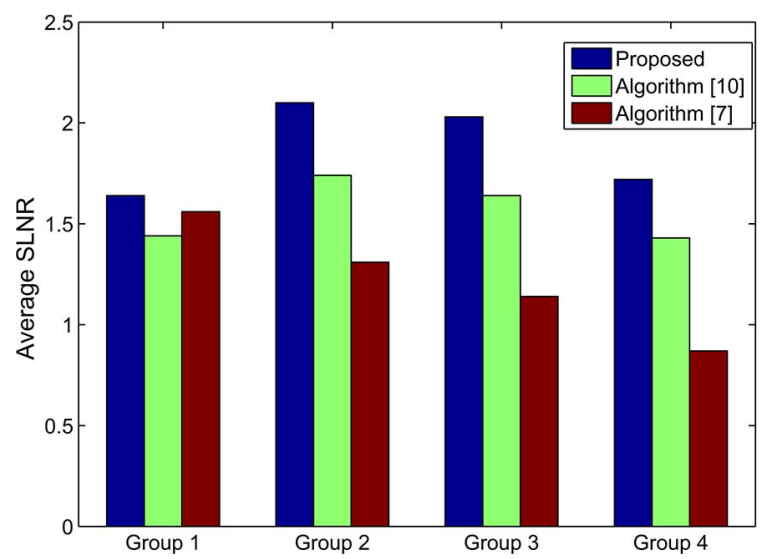

Fig. 5. Average SLNR versus each group for various algorithms $(M=128$, $G=4, M_{g}=32, K_{g}=S_{g}=5, \Delta=\pi / 13, P_{T}=15 \mathrm{~dB}$, and $\theta=-45^{\circ}$, $-15^{\circ}, 15,^{\circ}$ and $45^{\circ}$ ).

matrix setup to see the impact of a fixed weighting factor. The channel covariance matrix is a function of the center angle and angular spread according to the model (2). We fixed the center angles of four virtual subsectors as $\theta=-45^{\circ},-15^{\circ},-15^{\circ}$, and $45^{\circ}$ but varied AS $\Delta$. Fig. 4 shows the average sum rate performance with respect to $\Delta$ when the transmit power is $-10 \mathrm{~dB}$. (Note the transmit power $-10 \mathrm{~dB}$ point in Fig. 2.) It is seen that at a certain $\mathrm{AS}$, the fixed weighting factor $w=$ 1 yields good performance but deteriorated performance for different AS's. Thus, the optimization of the weighting factor depending on different channel statistics is necessary for good performance for arbitrary channel statistics.

Fig. 5 shows the average SLNR performance for each group under the same setting as in Fig. 2. The shown values are average values over 2000 channel realizations. It is seen that the proposed algorithm outperforms the other two algorithms. The algorithm in [7] has relatively poor performance compared to the other algorithms since it only considers the subspace spanned by the dominant eigenvectors of each channel covariance matrix. The channel component generated by the nondominant eigenvectors can result in nonnegligible interference.
Moreover, the algorithm in [7] is based on the sequential projection from Group 1 to Group 4. Thus, the effective channel power for each group is degraded at every projection step, and this is clearly shown in Fig. 5.

Finally, we tested the convergence of the proposed algorithm. Fig. (6)(a) shows the objective value $\rho_{n}$ of the TQP for each group by the proposed algorithm as a function of iteration time. It is seen that the proposed algorithm monotonically converges, as expected from Theorem 2, and furthermore the proposed algorithm converges after only a few iterations. Next, we tested the convergence of the proposed algorithm with different initial points. Fig. 6(b) shows the objective value $\rho_{n}$ of the TQP for Group 1 for different initial points. It is seen that the algorithm with the proposed initial point based on (25) converges much faster than the other two random initial points. Since the proposed algorithm requires eigenvalue decomposition (EVD) for each iteration step, the number of required iterations directly translates into computational complexity. Thus, the initialization based on (25) reduces computational complexity significantly compared to random initialization, although the algorithm with a random initial point also converges eventually.

\section{Conclusion}

In this paper, we have considered the outer beamformer design problem based only on channel statistic information for two-stage beamforming for massive MIMO downlink and have proposed an outer beamformer design method which maximizes a lower bound on the average SLNR. We have shown that the proposed SLNR-based outer beamformer design problem reduces to a TQP, which is often encountered in the field of machine learning, and have presented an iterative algorithm to obtain an optimal solution to the proposed TQP. Numerical results show that the proposed outer beamformer design method yields significant performance gain over existing methods. The proposed method can easily be adapted to the multi-cell scenario in which each base station serves one group of users.

\section{APPENDIX A \\ PROOF OF THEOREM 1}

Using similar techniques to those used in the proof of Lemma 1 in [28] and the proof of Theorem 1 in [11], we first prove several lemmas necessary for proof of Theorem 1.

Lemma 1: For ZF inner beamforming, we have

$$
\left|\mathbf{h}_{g_{k}}^{H} \mathbf{V}_{g} \mathbf{w}_{g_{k}}\right|^{2}=\mathbf{h}_{g_{k}}^{H} \mathbf{V}_{g} \widetilde{\mathbf{U}}_{g_{k}} \widetilde{\mathbf{U}}_{g_{k}}^{H} \mathbf{V}_{g}^{H} \mathbf{h}_{g_{k}}
$$

where the column space of $\widetilde{\mathbf{U}}_{g_{k}}$ is the null space of the composite effective channel matrix $\widetilde{\mathbf{H}}_{g,-k}$ except the channel of user $g_{k}$, i.e., $\widetilde{\mathbf{H}}_{g,-k} \triangleq\left[\mathbf{V}_{g}^{H} \mathbf{h}_{g_{1}}, \cdots, \mathbf{V}_{g}^{H} \mathbf{h}_{g_{k-1}}, \mathbf{V}_{g}^{H} \mathbf{h}_{g_{k+1}} \cdots \mathbf{V}_{g}^{H} \mathbf{h}_{g_{K_{g}}}\right]^{H}$.

Proof: $\quad \widetilde{\mathbf{H}}_{g,-k} \in \mathcal{C}^{\left(K_{g}-1\right) \times M_{g}}\left(K_{g} \leq M_{g}\right)$ is decomposed by singular value decomposition (SVD) as

$$
\widetilde{\mathbf{H}}_{g,-k}=\boldsymbol{\Upsilon}_{g_{k}} \mathbf{D}_{g_{k}}\left[\begin{array}{c}
\mathbf{U}_{g_{k}}^{H} \\
\widetilde{\mathbf{U}}_{g_{k}}^{H}
\end{array}\right],
$$




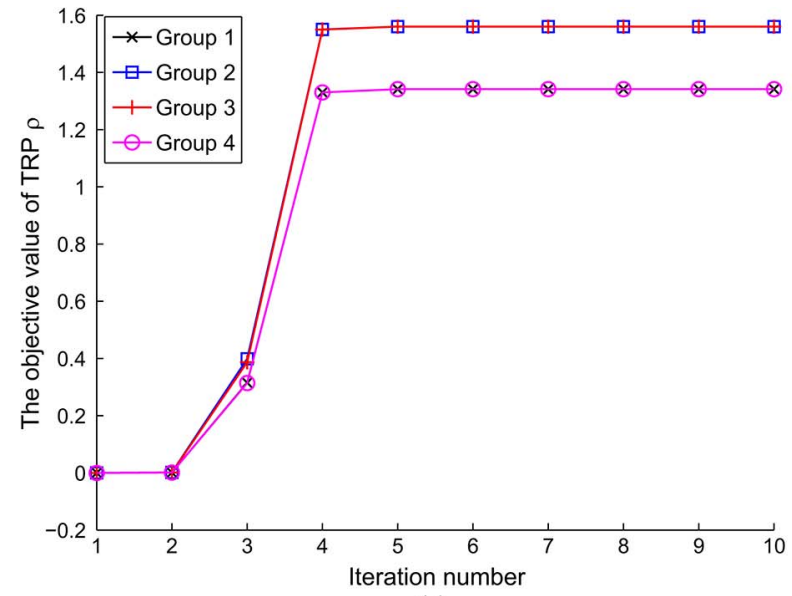

(a)

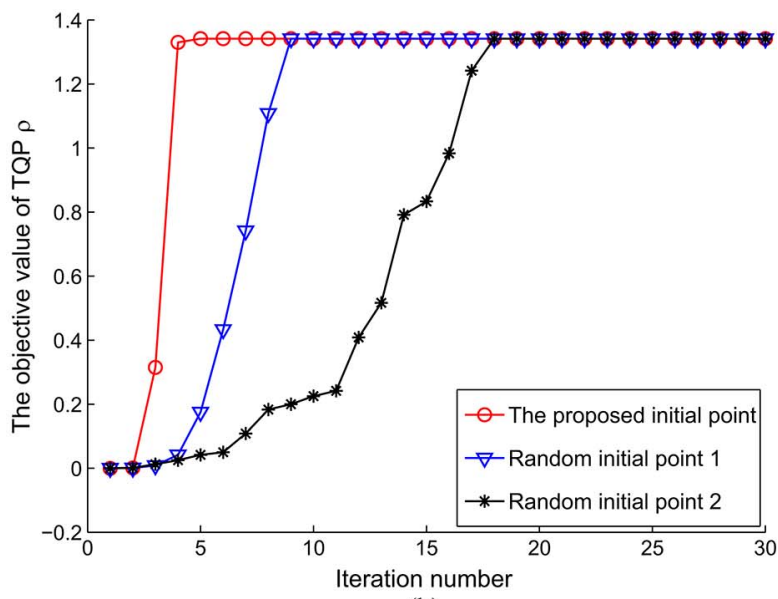

(b)

Fig. 6. (a) The objective value of the TQP for each group versus iteration number and (b) the objective value of the TQP of Group 1 for various initial points versus iteration number $\left(M=128, G=4, M_{g}=32, K_{g}=S_{g}=5, \Delta=\pi / 13\right.$, and $\theta=-45^{\circ},-15^{\circ}, 15^{\circ}$, and $\left.45^{\circ}\right)$.

where $\Upsilon_{g_{k}}$ is a $\left(K_{g}-1\right) \times\left(K_{g}-1\right)$ unitary matrix, $\mathbf{D}_{g_{k}}$ is a $M_{g} \times M_{g}$ diagonal matrix, $\mathbf{U}_{g_{k}}$ is a $M_{g} \times\left(K_{g}-1\right)$ submatrix whose column space is the row space of $\widetilde{\mathbf{H}}_{g,-k}$, and $\widetilde{\mathbf{U}}_{g_{k}}$ is a $M_{g} \times\left(M_{g}-K_{g}+1\right)$ submatrix whose column space is the null space of $\widetilde{\mathbf{H}}_{g,-k}$. The ZF inner beamformer $\mathbf{w}_{g_{k}}$ with equal power can be expressed by the projection of the effective channel $\mathbf{V}_{g}^{H} \mathbf{h}_{g_{k}}$ of user $g_{k}$ to $\widetilde{\mathbf{U}}_{g_{k}}$ as [28]

$$
\mathbf{w}_{g_{k}}=\frac{\widetilde{\mathbf{U}}_{g_{k}} \tilde{\mathbf{U}}_{g_{k}}^{H} \mathbf{V}_{g}^{H} \mathbf{h}_{g_{k}}}{\left\|\widetilde{\mathbf{U}}_{g_{k}} \tilde{\mathbf{U}}_{g_{k}}^{H} \mathbf{V}_{g}^{H} \mathbf{h}_{g_{k}}\right\|} .
$$

By (38), $\left|\mathbf{h}_{g_{k}}^{H} \mathbf{V}_{g} \mathbf{w}_{g_{k}}\right|^{2}$ is expressed as

$$
\begin{aligned}
\left|\mathbf{h}_{g_{k}}^{H} \mathbf{V}_{g} \mathbf{w}_{g_{k}}\right|^{2} & =\mathbf{h}_{g_{k}}^{H} \mathbf{V}_{g} \mathbf{w}_{g_{k}} \mathbf{w}_{g_{k}}^{H} \mathbf{V}_{g}^{H} \mathbf{h}_{g_{k}}, \\
& =\frac{\mathbf{h}_{g_{k}}^{H} \mathbf{V}_{g} \widetilde{\mathbf{U}}_{g_{k}} \widetilde{\mathbf{U}}_{g_{k}}^{H} \mathbf{V}_{g}^{H} \mathbf{h}_{g_{k}} \mathbf{h}_{g_{k}}^{H} \mathbf{V}_{g} \widetilde{\mathbf{U}}_{g_{k}} \widetilde{\mathbf{U}}_{g_{k}}^{H} \mathbf{V}_{g}^{H} \mathbf{h}_{g_{k}}}{\left\|\widetilde{\mathbf{U}}_{g_{k}} \widetilde{\mathbf{U}}_{g_{k}}^{H} \mathbf{V}_{g}^{H} \mathbf{h}_{g_{k}}\right\|^{2}} \\
& =\mathbf{h}_{g_{k}}^{H} \mathbf{V}_{g} \widetilde{\mathbf{U}}_{g_{k}} \widetilde{\mathbf{U}}_{g_{k}}^{H} \mathbf{V}_{g}^{H} \mathbf{h}_{g_{k}} .
\end{aligned}
$$

This concludes the proof.

Lemma 2: The conditional expectation of $\left\|\mathbf{h}_{g_{k}}^{H} \mathbf{V}_{g} \mathbf{w}_{g_{k}}\right\|^{2}$ for given $\widetilde{\mathbf{H}}_{g,-k}$ is given by

$$
\mathbb{E}\left\{\left|\mathbf{h}_{g_{k}}^{H} \mathbf{V}_{g} \mathbf{w}_{g_{k}}\right|^{2} \mid \widetilde{\mathbf{H}}_{g,-k}\right\}=\operatorname{Tr}\left(\boldsymbol{\Sigma}_{g_{k}}\right),
$$

where

$$
\boldsymbol{\Sigma}_{g_{k}}=\widetilde{\mathbf{U}}_{g_{k}}^{H} \mathbf{V}_{g}^{H} \mathbf{R}_{g} \mathbf{V}_{g} \widetilde{\mathbf{U}}_{g_{k}}
$$

and $\widetilde{\mathbf{U}}_{g_{k}}$ is defined in (37) in Lemma 1.

Proof: By Lemma 1, we have $\left|\mathbf{h}_{g_{k}}^{H} \mathbf{V}_{g} \mathbf{w}_{g_{k}}\right|^{2}=\mathbf{h}_{g_{k}}^{H} \mathbf{V}_{g} \widetilde{\mathbf{U}}_{g_{k}} \widetilde{\mathbf{U}}_{g_{k}}^{H}$ $\mathbf{V}_{g}^{H} \mathbf{h}_{g_{k}}$. For given $\widetilde{\mathbf{H}}_{g,-k}, \widetilde{\mathbf{U}}_{g_{k}}$ is also given because $\widetilde{\mathbf{U}}_{g_{k}}$ deterministically depends on $\widetilde{\mathbf{H}}_{g,-k}$ according to (37). Define $\boldsymbol{\xi}_{g_{k}} \triangleq$ $\widetilde{\mathbf{U}}_{g_{k}}^{H} \mathbf{V}_{g}^{H} \mathbf{h}_{g_{k}}$. Then, $\boldsymbol{\xi}_{g_{k}} \mid \widetilde{\mathbf{H}}_{g,-k}$ is a zero-mean complex Gaussian random vector with the covariance matrix $\boldsymbol{\Sigma}_{g_{k}}=\widetilde{\mathbf{U}}_{g_{k}}^{H} \mathbf{V}_{g}^{H} \mathbf{R}_{g} \mathbf{V}_{g}$
$\widetilde{\mathbf{U}}_{g_{k}}$, i.e., $\boldsymbol{\xi}_{g_{k}} \mid \widetilde{\mathbf{H}}_{g,-k} \sim \mathcal{C N}\left(0, \boldsymbol{\Sigma}_{g_{k}}\right)$, since $\mathbf{h}_{g_{k}} \sim \mathcal{C N}\left(0, \mathbf{R}_{g}\right)$ from (1). Let the eigenvalue decomposition of $\boldsymbol{\Sigma}_{g_{k}}$ be

$$
\boldsymbol{\Sigma}_{g_{k}}=\overline{\mathbf{U}}_{g_{k}} \overline{\mathbf{\Lambda}}_{g_{k}} \overline{\mathbf{U}}_{g_{k}}^{H}
$$

where $\overline{\boldsymbol{\Lambda}}_{g_{k}}=\operatorname{diag}\left(\bar{\lambda}_{g_{k}, 1}, \cdots, \bar{\lambda}_{g_{k}, N_{g}}\right)$ and $N_{g}=M_{g}-K_{g}+1$. Then, $\boldsymbol{\xi}_{g_{k}}$ can be expressed as

$$
\boldsymbol{\xi}_{g_{k}} \mid \widetilde{\mathbf{H}}_{g,-k}=\Sigma_{g_{k}}^{1 / 2} \boldsymbol{\eta}=\overline{\mathbf{U}}_{g_{k}} \overline{\boldsymbol{\Lambda}}_{g_{k}}^{1 / 2} \boldsymbol{\eta}
$$

where $\boldsymbol{\eta} \sim \mathcal{C N}\left(0, \mathbf{I}_{M_{g}-K_{g}+1}\right)$. Thus, $\left|\mathbf{h}_{g_{k}}^{H} \mathbf{V}_{g} \mathbf{w}_{g_{k}}\right|^{2}$ can be rewritten as

$$
\begin{aligned}
\left|\mathbf{h}_{g_{k}}^{H} \mathbf{V}_{g} \mathbf{w}_{g_{k}}\right|^{2} & =\mathbf{h}_{g_{k}}^{H} \mathbf{V}_{g} \widetilde{\mathbf{U}}_{g_{k}} \widetilde{\mathbf{U}}_{g_{k}}^{H} \mathbf{V}_{g}^{H} \mathbf{h}_{g_{k}}, \\
& =\boldsymbol{\xi}_{g_{k}}^{H} \boldsymbol{\xi}_{g_{k}}=\operatorname{Tr}\left(\boldsymbol{\xi}_{g_{k}} \boldsymbol{\xi}_{g_{k}}^{H}\right), \\
& =\operatorname{Tr}\left(\overline{\mathbf{U}}_{g_{k}} \overline{\boldsymbol{\Lambda}}_{g_{k}}^{1 / 2} \boldsymbol{\eta} \boldsymbol{\eta}^{H} \overline{\boldsymbol{\Lambda}}_{g_{k}}^{H / 2} \overline{\mathbf{U}}_{g_{k}}^{H}\right) .
\end{aligned}
$$

Using (41), we now take the conditional expectation of $\left|\mathbf{h}_{g_{k}}^{H} \mathbf{V}_{g} \mathbf{w}_{g_{k}}\right|^{2}$ for given $\widetilde{\mathbf{H}}_{g,-k}$ :

$$
\begin{aligned}
\mathbb{E}\left\{\left|\mathbf{h}_{g_{k}}^{H} \mathbf{V}_{g} \mathbf{w}_{g_{k}}\right|^{2} \mid \widetilde{\mathbf{H}}_{g,-k}\right\} \\
=\mathbb{E}\left\{\operatorname{Tr}\left(\overline{\mathbf{U}}_{g_{k}} \overline{\boldsymbol{\Lambda}}_{g_{k}}^{1 / 2} \boldsymbol{\eta} \boldsymbol{\eta}^{H} \overline{\boldsymbol{\Lambda}}_{g_{k}}^{H / 2} \overline{\mathbf{U}}_{g_{k}}^{H}\right) \mid \widetilde{\mathbf{H}}_{g,-k}\right\}, \\
=\operatorname{Tr}\left(\overline{\mathbf{U}}_{g_{k}} \overline{\boldsymbol{\Lambda}}_{g_{k}}^{1 / 2} \mathbb{E}\left\{\boldsymbol{\eta} \boldsymbol{\eta}^{H} \mid \widetilde{\mathbf{H}}_{g,-k}\right\} \overline{\boldsymbol{\Lambda}}_{g_{k}}^{H / 2} \overline{\mathbf{U}}_{g_{k}}^{H}\right) \\
=\operatorname{Tr}\left(\overline{\mathbf{U}}_{g_{k}} \overline{\boldsymbol{\Lambda}}_{g_{k}}^{1 / 2} \overline{\boldsymbol{\Lambda}}_{g_{k}}^{H / 2} \overline{\mathbf{U}}_{g_{k}}^{H}\right), \\
=\sum_{i=1}^{N_{g}} \overline{\boldsymbol{\lambda}}_{g_{k}, i}=\operatorname{Tr}\left(\boldsymbol{\Sigma}_{g_{k}}\right) .
\end{aligned}
$$

Lemma 3: [25] [Corollary 4.3.18] Let $\mathbf{M}$ be any $J \times J$ Hermitian matrix and let $j$ be a given integer such that $1 \leq j \leq J$. Then, we have

$$
\lambda_{1}+\lambda_{2}+\cdots+\lambda_{j}=\min _{\mathbf{U}^{H} \mathbf{U}=\mathbf{I}_{j}} \operatorname{Tr}\left(\mathbf{U}^{H} \mathbf{M} \mathbf{U}\right),
$$

and

$$
\lambda_{J-j+1}+\lambda_{J-j+2}+\cdots+\lambda_{J}=\max _{\mathbf{U}^{H} \mathbf{U}=\mathbf{I}_{j}}\left(\mathbf{U}^{H} \mathbf{M U}\right),
$$


where $\lambda_{k}$ is the $k$-th smallest eigenvalue of $\mathbf{M}$ and equality holds if the columns of the $J \times j$ matrix $\mathbf{U}$ are chosen to be the orthonormal eigenvectors associated with the corresponding eigenvalues of $\mathbf{M}$.

Lemma 4: For the matrix $\boldsymbol{\Sigma}_{g_{k}}$ defined in Lemma 2, $\left(\boldsymbol{\Sigma}_{g_{k}}\right)$ is lower bounded as

$$
\operatorname{Tr}\left(\boldsymbol{\Sigma}_{g_{k}}\right) \geq \operatorname{Tr}\left(\mathbf{V}_{g}^{H} \mathbf{R}_{g} \mathbf{V}_{g}\right)-\left(K_{g}-1\right) \lambda_{g},
$$

where $\mathbf{V}_{g}$ is the outer beamformer for group $g$ satisfying $\mathbf{V}_{g}^{H} \mathbf{V}_{g}=\mathbf{I}, \mathbf{R}_{g}$ is the channel covariance matrix of group $g$, and $\lambda_{g}$ is the largest eigenvalue of $\mathbf{R}_{g}$.

Proof: Applying the Rayleigh quotient technique, one can easily show that the maximum eigenvalue of the matrix $\mathbf{V}_{g}^{H} \mathbf{R}_{g} \mathbf{V}_{g}$ is less than or equal to $\lambda_{g}$, where $\lambda_{g}$ is the largest eigenvalue of $\mathbf{R}_{g}$, since $\mathbf{V}_{g}$ has orthonormal columns. That is,

$$
\begin{aligned}
\lambda_{\max }\left(\mathbf{V}_{g}^{H} \mathbf{R}_{g} \mathbf{V}_{g}\right) & \leq \max \frac{\mathbf{x}^{H} \mathbf{V}_{g}^{H} \mathbf{R}_{g} \mathbf{V}_{g} \mathbf{x}^{H}}{\mathbf{x}^{H} \mathbf{x}}, \\
& =\max \frac{\mathbf{x}^{H} \mathbf{V}_{g}^{H} \mathbf{R}_{g} \mathbf{V}_{g} \mathbf{x}^{H}}{\mathbf{x}^{H} \mathbf{V}_{g}^{H} \mathbf{V}_{g} \mathbf{x}}, \\
& =\max \frac{\tilde{\mathbf{x}}^{H} \mathbf{R}_{g} \tilde{\mathbf{x}}^{H}}{\tilde{\mathbf{x}}^{H} \tilde{\mathbf{x}}}, \\
& =\lambda_{g} .
\end{aligned}
$$

Define $\boldsymbol{\Pi}_{g} \triangleq \frac{1}{\lambda_{g}} \mathbf{V}_{g}^{H} \mathbf{R}_{g} \mathbf{V}_{g}$ and let $0 \leq \chi_{1} \leq \chi_{2} \leq \cdots, \chi_{M_{g}}$ be the eigenvalues of $\Pi_{g}$. Then, the maximum eigenvalue $\chi_{M_{g}}$ of $\boldsymbol{\Pi}_{g}$ is less than or equal to one from the above. Note from (40) that $\frac{1}{\lambda_{g}} \boldsymbol{\Sigma}_{g_{k}}=\widetilde{\mathbf{U}}_{g_{k}}^{H} \boldsymbol{\Pi}_{g} \widetilde{\mathbf{U}}_{g_{k}}$, where $\widetilde{\mathbf{U}}_{g_{k}}^{H} \widetilde{\mathbf{U}}_{g_{k}}=\mathbf{I}$. Applying Lemma 3 to $\frac{1}{\lambda_{g}} \boldsymbol{\Sigma}_{g_{k}}$ with $\mathbf{M}=\boldsymbol{\Pi}_{g}$, we have

$$
\begin{aligned}
\frac{1}{\lambda_{g}} \operatorname{Tr}\left(\boldsymbol{\Sigma}_{g_{k}}\right) & \geq \sum_{k=1}^{N_{g}} \chi_{k}, \\
& \stackrel{(a)}{\geq} \sum_{k=1}^{M_{g}} \chi_{k}-\left(K_{g}-1\right) \chi_{M_{g}}, \\
& \stackrel{(b)}{\geq} \sum_{k=1}^{M_{g}} \chi_{k}-\left(K_{g}-1\right), \\
& =\operatorname{Tr}\left(\boldsymbol{\Pi}_{g}\right)-\left(K_{g}-1\right),
\end{aligned}
$$

where (a) follows from the relationship $N_{g}=M_{g}-K_{g}+1$ and the fact that $\chi_{M_{g}}$ is the maximum eigenvalue of $\Pi_{g}$ and (b) follows from the fact that $\chi_{M_{g}} \leq 1$. Multiplying both sides of (49) by $\lambda_{g}$, we obtain the desired result.

Proof of Theorem 1: Finally, we prove Theorem 1 by using the above lemmas. By Lemma 2 and Lemma 4, the conditional expectation of $\left|\mathbf{h}_{g_{k}}^{H} \mathbf{V}_{g} \mathbf{w}_{g_{k}}\right|^{2}$ for given $\tilde{\mathbf{H}}_{g,-k}$ is lowerbounded as

$$
\begin{aligned}
\mathbb{E}\left\{\left|\mathbf{h}_{g_{k}}^{H} \mathbf{V}_{g} \mathbf{w}_{g_{k}}\right|^{2} \mid \widetilde{\mathbf{H}}_{g,-k}\right\} & =\operatorname{Tr}\left(\boldsymbol{\Sigma}_{g_{k}}\right), \\
& \geq \operatorname{Tr}\left(\mathbf{V}_{g}^{H} \mathbf{R}_{g} \mathbf{V}_{g}\right)-\left(K_{g}-1\right) \lambda_{g},
\end{aligned}
$$

where (50) is by Lemma 2 and (51) is by Lemma 4 . Note that the lower bound (51) is independent of $\widetilde{\mathbf{H}}_{g,-k}$ and is a constant.
By taking expectation over $\widetilde{\mathbf{H}}_{g,-k}$ on both sides of (51). The left-hand side (LHS) becomes $\mathbb{E}\left\{\left|\mathbf{h}_{g_{k}}^{H} \mathbf{V}_{g} \mathbf{w}_{g_{k}}\right|^{2}\right\}$ by the law of iterated expectation and the RHS does not change since the RHS is a constant. Hence, we have

$$
\mathbb{E}\left\{\left|\mathbf{h}_{g_{k}}^{H} \mathbf{V}_{g} \mathbf{w}_{g_{k}}\right|^{2}\right\} \geq \operatorname{Tr}\left(\mathbf{V}_{g}^{H} \mathbf{R}_{g} \mathbf{V}_{g}\right)-\left(K_{g}-1\right) \lambda_{g} .
$$

\section{APPENDIX B}

\section{PROOF OF THEOREM 2}

As in the proof of Lemma 1 in [17], we first define $f_{n}\left(\mathbf{V}_{g}\right) \triangleq$ $\operatorname{Tr}\left(\mathbf{V}_{g}{ }^{H}\left(\mathbf{R}_{g, 1}-\rho_{n} \mathbf{R}_{g, 2}\right) \mathbf{V}_{g}\right)$. Then, we have $f_{n}\left(\mathbf{V}_{g}^{(n-1)}\right)=0$ from (27). The step (30) of Algorithm 1 maximizes $f_{n}\left(\mathbf{V}_{g}\right)$ over the set $\left\{\mathbf{V}_{g}: \mathbf{V}_{g}^{H} \mathbf{V}_{g}=\mathbf{I}\right\}$ which includes $\mathbf{V}_{g}^{(n-1)}$, and $\mathbf{V}_{g}^{(n)}$ is the maximizer of $f_{n}\left(\mathbf{V}_{g}\right)$. Hence, we have

$$
f_{n}\left(\mathbf{V}_{g}^{(n)}\right) \geq f_{n}\left(\mathbf{V}_{g}^{(n-1)}\right)=0 .
$$

Based on the positive-definiteness of $\mathbf{R}_{g, 2}, f_{n}\left(\mathbf{V}_{g}^{(n)}\right)=$ $\left(\mathbf{V}_{g}^{(n)}{ }^{H}\left(\mathbf{R}_{g, 1}-\rho_{n} \mathbf{R}_{g, 2}\right) \mathbf{V}_{g}^{(n)}\right) \geq 0$ can be rewritten as

$$
\rho_{n+1}=\frac{\operatorname{Tr}\left(\mathbf{V}_{g}^{(n)^{H}} \mathbf{R}_{g, 1} \mathbf{V}_{g}^{(n)}\right)}{\operatorname{Tr}\left(\mathbf{V}_{g}^{(n)}{ }^{H} \mathbf{R}_{g, 2} \mathbf{V}_{g}^{(n)}\right)} \geq \rho_{n} .
$$

This concludes the proof.

\section{APPENDIX C PROOF OF THEOREM 3}

By Lemma 3, the numerator and denominator of the objective function of TQP are lower-bouned and upper-bounded as

$$
\begin{gathered}
\sum_{k=1}^{M_{g}} \psi_{M-k} \leq \operatorname{Tr}\left(\mathbf{V}_{g}^{H} \mathbf{R}_{g, 1} \mathbf{V}_{g}\right) \leq \sum_{k=1}^{M_{g}} \psi_{k} \text { and } \\
\sum_{k=1}^{M_{g}} \varphi_{M-k} \leq \operatorname{Tr}\left(\mathbf{V}_{g}^{H} \mathbf{R}_{g, 2} \mathbf{V}_{g}\right) \leq \sum_{k=1}^{M_{g}} \varphi_{k} .
\end{gathered}
$$

$\mathbf{R}_{g, 1}$ is Hermitian and $\mathbf{R}_{g, 2}$ is positive-definite. Hence, we have $\sum_{k=1}^{M_{g}} \varphi_{M-k}>0$ and $\sum_{k=1}^{M_{g}} \varphi_{k}>0$. We consider three cases: i) $\sum_{k=1}^{M_{g}} \psi_{M-k} \geq 0$ and $\sum_{k=1}^{M_{g}} \psi_{k} \geq 0$, ii) $\sum_{k=1}^{M_{g}} \psi_{M-k}<0$ and $\sum_{k=1}^{M_{g}} \psi_{k} \geq 0$, and iii) $\sum_{k=1}^{M_{g}} \psi_{M-k}<0$ and $\sum_{k=1}^{M_{g}} \psi_{k}<0$. By applying (55) to each case, we have the desired result.

\section{REFERENCES}

[1] T. L. Marzetta, "Noncooperative cellular wireless with unlimited numbers of base station antennas," IEEE Trans. Wireless Commun., vol. 9, no. 11, pp. 3590-3600, Nov. 2010.

[2] F. Rusek et al., "Scaling up MIMO: Opportunities and challenges with very large arrays," IEEE Signal Process. Mag., vol. 30, no. 1, pp. 40-60, Jan. 2013

[3] S. Noh, M. D. Zoltowski, Y. Sung, and D. J. Love, "Pilot beam pattern design for channel estimation in massive MIMO systems," IEEE J. Sel. Topics Signal Process., vol. 8, no. 5, pp. 787-801, Oct. 2014.

[4] J. Choi, D. J. Love, and P. Bidigare, "Downlink training techniques for FDD massive MIMO systems: Open-loop and closed-loop training with 
memory," IEEE J. Sel. Topics Signal Process., vol. 8, no. 5, pp. 802-814, Oct. 2014.

[5] J. So, D. Kim, Y. Lee, and Y. Sung, "Pilot signal design for massive MIMO systems: A received signal-to-noise-ratio-based approach," IEEE Signal Process. Lett., vol. 22, no. 5, pp. 549-553, May 2015.

[6] S. Noh, M. Zoltowski, and D. Love, "Training sequence design for feedback assisted hybrid beamforming in massive MIMO systems," IEEE Trans. Commun., submitted for publication, 2015. [Online]. Available: http://arxiv.org/pdf/1407.1786v1.pdf

[7] A. Adhikary, J. Nam, J. Ahn, and G. Caire, "Joint spatial division and multiplexing-The large-scale array regime," IEEE Trans. Inf. Theory, vol. 59, no. 10, pp. 6641-6463, Oct. 2013.

[8] J. Nam, A. Adhikary, J. Ahn, and G. Caire, "Joint spatial division and multiplexing: Opportunistic beamforming, user grouping and simplified downlink scheduling," IEEE J. Sel. Topics Signal Process., vol. 8, no. 5, pp. 876-890, Oct. 2014.

[9] G. Lee and Y. Sung, "A new approach to user scheduling in massive multiuser MIMO broadcast channels," IEEE Trans. Inf. Theory, submitted for publication. [Online]. Available: http://arxiv.org/pdf/1403.6931.pdf

[10] J. Chen and V. Lau, "Two-tier precoding for FDD multi-cell massive MIMO time-varying interference networks," IEEE J. Sel. Areas Commun., vol. 32 , no. 6, pp. 1230-1238, Jun. 2014

[11] A. Liu and V. Lau, "Phase only RF precoding for massive MIMO systems with limited RF chains," IEEE Trans. Signal Process., vol. 62, no. 17, pp. 4505-4515, Sep. 2014.

[12] M. Sadek, A. Tarighat, and A. H. Sayed, "A leakage-based precoding scheme for downlink multi-user MIMO channels," IEEE Trans. Wireless Commun., vol. 6, no. 5, pp. 1711-1721, May 2007.

[13] R. Zakhour and D. Gesbert, "Coordination on the MISO interference channel using the virtual SINR framework," in Proc. Int. ITG Workshop Smart Antennas, Berlin, Germany, Feb. 2009, pp. 1-7.

[14] J. Park, G. Lee, Y. Sung, and M. Yukawa, "Coordinated beamforming with relaxed zero forcing: The sequential orthogonal projection combining method and rate control," IEEE Trans. Signal Process., vol. 61, no. 12, pp. 3100-3112, Jun. 2013.

[15] H. Shen, K. Diepold, and K. Hüper, "A geometric revisit to the trace quotient problem," in Proc. Int. Symp. MTNS, 2010, pp. 1-4.

[16] L. Zhang, W. Yang, and L. Liao, "A note on the trace quotient problem," Optim. Lett., vol. 8, no. 5, pp. 1637-1645, Jun. 2014.

[17] H. Wang, S. Yan, D. Xu, X. Tang, and T. Huang, "Trace ratio vs. ratio trace for dimensionality reduction," in Proc. IEEE Int. Conf. CVPR, 2007, pp. $136-139$.

[18] J. H. Kotecha and A. M. Sayeed, "Transmit signal design for optimal estimation of correlated MIMO channels," IEEE Trans. Signal Process., vol. 52, no. 2, pp. 546-557, Feb. 2004.

[19] A. Abdi and M. Kaveh, "A space-time correlation model for multielement antenna systems in mobile fading channels," IEEE J. Sel. Areas Commun., vol. 20, no. 3, pp. 550-560, Apr. 2002.

[20] M. Zhang, P. J. Smith, and M. Shafi, "An extended one-ring MIMO channel model," IEEE Trans. Wireless Commun., vol. 6, no. 8, pp. 2759-2764, Aug. 2007.

[21] G. H. Golub and C. F. V. Loan, Matrix Computations, 2nd ed. Baltimore, MD, USA: The Johns Hopkins Univ. Press, 1996.

[22] R.-C. Li, "Rayleigh quotient based numerical methods for eigenvalue problems," The Fourth Gene Golub SIAM Summer School, Fudan University, Shanghai, China, Jul. 2013.

[23] L. Zhang, L. Liao, and M. Ng, "Fast algorithms for the generalized Foley-Sammon discriminant analysis," SIAM J. Matrix Anal. Appl., vol. 31, no. 4, pp. $1584-1605,2010$.

[24] B. N. Parlett, The Symmetric Eigenvalue Problem. Philadelphia, PA, USA: SIAM, 1998.
[25] R. A. Horn and C. R. Johnson, Matrix Analysis. Cambridge, U.K.: Cambridge Univ. Press, 1987.

[26] C. B. Peel, B. M. Hochwald, and A. L. Swindlehurst, "A vectorperturbation technique for near-capacity multiantenna multiuser communication-Part I: Channel inversion and regularization," IEEE Trans. Commun., vol. 53, no. 1, pp. 195-202, Jan. 2005.

[27] M. Joham, K. Kusume, M. H. Gzara, and W. Utschick, "Transmit Wiener filter for the downlink of TDD DS-CDMA systems," in Proc. IEEE 7th Symp. Spread-Spectrum Technol. Appl., Prague, Czech Republic, Sep. 2002, pp. 136-139.

[28] P. Li, D. Paul, R. Narasimhan, and J. Cioffi, "On the distribution of SINR for the MMSE MIMO receiver and performance analysis," IEEE Trans. Inf. Theory, vol. 52, no. 1, pp. 271 - 286, Jan. 2006.

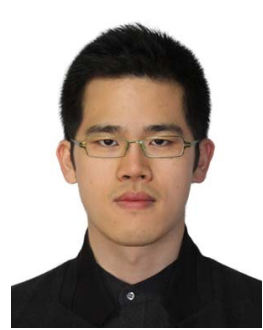

Donggun Kim (S'10) received the B.S. degree in electronics and communications engineering from Hanyang University, Seoul, Korea, in 2010. He is currently working toward the Ph.D. degree in the Department of Electrical Engineering, KAIST. His research interests include convex optimization, matrix theory, and signal processing for next generation wireless communications.

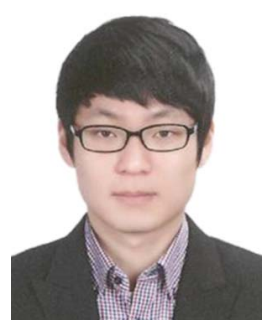

Gilwon Lee (S'10) received the B.S. and M.S degrees in electrical engineering from KAIST, Daejeon, South Korea, in 2010 and 2012, respectively. He is currently with the wireless information systems research group, KAIST, working towards the Ph.D. degree. His research interests are in the design and analysis of large-scale MIMO systems and signal processing for next wireless communications.

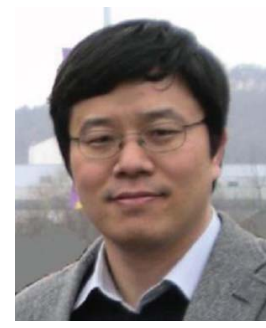

Youngchul Sung (S'92-M'93-SM'09) received the B.S. and M.S. degrees from Seoul National University, Seoul, Korea, in electronics engineering, in 1993 and 1995, respectively. After working at LG Electronics, Ltd., Seoul, Korea, from 1995 to 2000 , he joined the Ph.D. program and received the Ph.D. degree in electrical and computer engineering from Cornell University, Ithaca, NY, USA, in 2005. From 2005 to 2007, he was a Senior Engineer in the Corporate R\&D Center of Qualcomm, Inc., San Diego, CA, USA, and participated in design of WCDMA base station modem. Since 2007, he has been on the faculty of the Department of Electrical Engineering in KAIST, Daejeon, Korea. His research interests include signal processing for communications, statistical signal processing, asymptotic statistics, and information geometry. Dr. Sung is currently a member of UNESCO/Netexplo University Advisory Board, Signal and Information Processing Theory and Methods (SIPTM) Technical Committee of Asia-Pacific Signal and Information Processing Association (APSIPA), Vice-Chair of IEEE ComSoc Asia-Pacific Board ISC, and was an Associate Editor of the IEEE SignAL PROCESSING LetTERS from 2012 to 2014. 